Graphs and Combinatorics (1996) 12: 97-137

\title{
Optimal Pairs of Incomparable Clouds in Multisets
}

\author{
Rudolf Ahlswede ${ }^{1}$ and Levon H. Khachatrian ${ }^{2}$ \\ 1 Universität Bielefeld, Fakultät für Mathematik, Postfach 100131, 33501 Bielefeld, \\ Germany \\ 2 Institute of Problems of Information and Automation, Armenian Academy of Sciences, \\ Erevan-44 P. Sevak str. 1, Rep. of Armenia, Visiting SFB 343
}

\begin{abstract}
We consider the partially ordered set $\left([k]^{n}, \leq\right)$, which is defined as $n$-th product of the chain $[k]=\{0,1,2, \ldots, k-1\}$, and study pairs $(A, B)$ of incomparable sets $A, B \subset[k]^{n}$, that is, $a \not b, a \geq b$ for all $a \in A, b \in B$ or (in short notation) $A \neq k=B$.

We are concerned with the growth of the functions $f_{n}:\left\{0,1, \ldots, k^{n}\right\} \rightarrow\left\{0,1, \ldots, k^{n}\right\}$, $n \in \mathbb{N}$, defined by $f_{n}(\alpha)=\max \left\{|B|: A, B \subset[k]^{n}\right.$ with $|A|=\alpha$ and $\left.A=k B\right\}$ and a characterisation of pairs $(A, B)$, which assume this bound.

In the previously studied case $k=2$ our results are considerably sharper than earlier results by Seymour, Hilton, Ahlswede and Zhang.
\end{abstract}

\section{Introduction, Basic Results and Problems}

Let us be given the partially ordered set $\mathscr{P}_{n}\left([k]^{n}, \leq\right)$, where $[k]=\{0,1,2, \ldots$, $k-1\}$ and $a=\left(a_{1}, a_{2}, \ldots, a_{n}\right) \leq b=\left(b_{1}, b_{2}, \ldots, b_{n}\right)$ iff $a_{t} \leq b_{t}$ for $t=1,2, \ldots, n$. In the terminology of our earlier work ([1], [8], [9]) we call a pair $(A, B)$ with $A, B \subset[k]^{n}$ a cloud-antichain of length 2 , if

$$
a \nless b, \quad a ¥ b \quad \text { for all } \quad a \in A, \quad b \in B .
$$

A short expression for (1.1) is: $A \supset \mathcal{B}$.

We denote the set of these pairs by $\mathscr{C} \mathscr{A} \mathscr{C}(n)$. The objects of our investigation are the functions $f_{n}:\left\{0,1, \ldots, k^{n}\right\} \rightarrow\left\{0,1, \ldots, k^{n}\right\}, n \in \mathbb{N}$, defined by

$$
f_{n}(\alpha)=\max \{|B|: \exists(A, B) \in \mathscr{C} \mathscr{A} \mathscr{C}(n) \text { with }|A|=\alpha\}
$$

and a characterization of pairs $(A, B)$ which are optimal, that is, assume this bound. We denote by $\mathcal{O}(n)$ the set of all those optimal pairs.

In case where we emphasize the dependence on parameter $k$ we also write $\mathscr{C} \mathscr{A} \mathscr{C}_{k}(n), \mathcal{O}_{k}(n), f_{n, k}(\alpha)$, etc. instead of $\mathscr{C} \mathscr{A} \mathscr{C}(n), \mathcal{O}(n), f_{n}(\alpha)$, etc.

Previous work is discussed in [9], where the best results prior to those in this paper can be found. They are all for the binary alphabet, i.e. $k=2$. Familiarity with this paper may be helpful but is not necessary for an understanding of the present results and proofs. We extend here first the key result of that paper to the case of general $k$. 
Theorem 1. For every $\gamma, 0 \leq \gamma \leq k^{n-2}$, a pair $(A, B) \in \mathcal{O}(n)$ with $|A|=\gamma$ exists, such that for some component all $a$ in $A$ have $a 0$ and for some other component all $a$ in A have a $k-1$.

From here we derive by an approach similar to (but not identical with) that of [9] the main recursion.

Theorem 2. For every $\gamma, 0 \leq \gamma \leq k^{n-2}$,

$$
\begin{aligned}
& \text { (i) } f_{n}(\gamma)=(k-1) k^{n-1}+k f_{n-2}(\gamma)-(k-1) \gamma \text {. } \\
& \text { (ii) } f_{n+2 s}(\gamma)=\left(k^{s}-1\right)\left(k^{n+s}-\gamma\right)+k^{s} f_{n}(\gamma) \text { for } s \geq 0 .
\end{aligned}
$$

The explicit characterization of all pairs $\left(\gamma, f_{n}(\gamma)\right)$ given in [9] does not seem to allow a reasonably simple extension to general $k$. Therefore results concerning aspects of this characterization problem are already of interest.

Theorem 8 of [1] states that in the case $k=2$ for $(A, B) \in \mathscr{C} \mathscr{A} \mathscr{C}(n)$

(a) $|A||B| \leq 2^{2 n-4}$

(b) $\min \{|A|,|B|\} \leq 2^{n-2}$

and that these bounds are best possible.

The key observation was that for $(A, B) \in \mathscr{C} \mathscr{A} \mathscr{C}(n)$ we have the disjointness properties $(A \wedge B) \cap(A \vee B)=\varnothing,(A \wedge B) \cap(A \cup B)=\varnothing,(A \vee B) \cap(A \cup B)=\varnothing$, and $A \cap B=\varnothing$.

Therefore

$$
|A|+|B|+|A \vee B|+|A \wedge B| \leq 2^{n}
$$

and since by the arithmetic-geometric means inequality

$$
(|A||B||A \vee B||A \wedge B|)^{1 / 4} \leq \frac{|A|+|B|+|A \vee B|+|A \wedge B|}{4}
$$

we get

$$
|A||B||A \vee B||A \wedge B| \leq\left(\frac{2^{n}}{4}\right)^{4} .
$$

Now we use the AD-inequality

$$
|A||B| \leq|A \vee B||A \wedge B| \quad(\text { see }[6])
$$

and get

$$
|A||B| \leq 2^{2 n-4} .
$$

(b) is an immediate consequence.

Inspection shows that the same derivation is valid for all $k$ and thus for $(A, B) \in \mathscr{C} \mathscr{A} \mathscr{C}_{k}(n)$

$$
|A||B| \leq\left(\frac{k}{2}\right)^{4} k^{2 n-4}
$$

This is tight only for even $k$.

In [9] the arithmetic-geometric means inequality was applied to two terms and so do we now for general $k$. Hence, 


$$
\begin{aligned}
k^{n} & \geq|A|+|B|+|A \wedge B|+|A \vee B| \\
& \geq|A|+|B|+2 \sqrt{|A \wedge B||A \vee B|} \\
& \geq|A|+|B|+2 \sqrt{|A||B|}=(\sqrt{|A|}+\sqrt{|B|})^{2}
\end{aligned}
$$

and therefore

$$
|A|^{1 / 2}+|B|^{1 / 2} \leq k^{n / 2}
$$

Theorem 3 of [9] states two consequences in case $k=2$ :

For all $l, 0 \leq l \leq 2^{n}$

(c) $f_{2 n}\left(l^{2}\right)=\left(2^{n}-l\right)^{2}$

(d) $f_{2 n+1}\left(2 l^{2}\right)=2\left(2^{n}-l\right)^{2}$.

Problem I. For every $k$ describe all $(A, B) \in \mathscr{C} \mathscr{A} \mathscr{C}_{k}(n)$ with equality in (1.8). In the terminology of $[10]$ this is an equality characterization problem.

Problem II. How does $f_{n, k}(\alpha)$ behave asymptotically in $k, n$, and $\alpha$ ? Next we try to generalize statements of type (a) and (b) to general $k$.

Problem III. Determine

$$
G(n)=\max _{(A, B) \in \mathscr{C} \mathcal{A}^{\mathcal{S}} \mathcal{G}_{k}(n)} \min (|A|,|B|) .
$$

\section{Problem IV. Determine}

$$
Q(n)=\max _{(A, B) \in \mathcal{G} \otimes^{\prime} \mathcal{G}_{k}(n)}|A||B|
$$

Finally we solve

Problem V. Let $\Delta$ be any integer, $-k^{n} \leq \Delta \leq k^{n}$, find $a_{\Delta}(n)=\max \{|A|:(A, B) \in$ $\left.\mathscr{C} \mathscr{A} \mathscr{C}_{k}(n),|B|=|A|+\Delta\right\}$.

We completely solve problems III and IV and provide partial results for the other problems.

\section{Auxiliary Results}

We use for the proofs of our Theorems 1 and 2 results of Daykin, Kleitman and West [5]. They are described in the abstract of [5]. Except for a reference to these Theorems in brackets, we literally repeat the main part of the abstract:

"Let $L$ be a lattice of divisors of an integer (isomorphically, a direct product of chains). We prove $|A||B| \leq|L||A \wedge B|$ for any $A, B \subset L$ where $|\cdot|$ denotes cardinality and $A \wedge B=\{a \wedge b: a \in A, b \in B\} .|A \wedge B|$ attains its minimum for fixed $|A|,|B|$ when $A$ and $B$ are ideals (Theorem 2). $|\cdot|$ can be replaced by certain other weight functions (Theorem 3 ). When the $n$ chains are of equal size $k$, the elements may be viewed as $n$-digit $k$-ary numbers. Then for fixed $|A|,|B|,|A \wedge B|$ is mini- 
mized when $A$ and $B$ are $|A|$ and $|B|$ smallest $n$-digit $k$-ary numbers written backwards and forwards, respectively (Theorem 4). ${ }^{1}|A \wedge B|$ for these sets is determined and bounded (Theorem 5)."

We don't need Theorem 3. Whereas Theorems 2, 4 are selfexplanatory, we have to give the details of Theorem 5 .

Theorem 5. [5] Suppose $L$ is a product of $n$ chains of size $k, 0 \leq \alpha \leq k^{n}, 0 \leq \beta \leq k^{n}$. Let $\mu_{k}(n, \alpha, \beta)=\min \{|A \wedge B|:|A|=\alpha,|B|=\beta\}$ and $\varepsilon_{k}(n, \alpha, \beta)=\mu_{k}(n, \alpha, \beta)-\frac{\alpha \beta}{k^{n}}$. If $p k^{n-1}<\alpha \leq(p+1) k^{n-1}$ and $\beta \equiv r \bmod k$, then

(i) $\mu_{k}(n, \alpha, \beta)=\mu_{k}\left(n-1, \alpha-p k^{n-1},\left\lceil\frac{\beta-p}{k}\right\rceil\right)+ \begin{cases}0 & p=0 \\ \sum_{j=0}^{p-1}\left\lceil\frac{\beta-j}{k}\right\rceil ; & p>0\end{cases}$

(ii) $\varepsilon_{k}(n, \alpha, \beta)=\varepsilon_{k}\left(n-1, \alpha-p k^{n-1},\left[\frac{\beta-p}{k}\right]\right)+ \begin{cases}r\left(1-\frac{\alpha}{k^{n}}\right) ; & 0 \leq r \leq p \\ (k-r) \frac{\alpha}{k^{n}} ; & p<r<k\end{cases}$

Furthermore,

$$
\begin{aligned}
\varepsilon_{k}\left(n, k^{n}-\alpha, k^{n}-\beta\right) & =\varepsilon_{k}(n, \alpha, \beta) \\
\mu_{k}\left(n, k^{n}-\alpha, k^{n}-\beta\right) & =\mu_{k}(n, \alpha, \beta)+k^{n}-\alpha-\beta
\end{aligned}
$$

and, finally,

$$
0 \leq \varepsilon_{k}(n, \alpha, \beta) \leq \frac{k n}{4}
$$

$$
\text { (Immediate from definitions and added for the ease of reference) }
$$

$$
\mu_{k}(n, 0, \beta)=0 ; \quad \mu_{k}\left(n, k^{n}, \beta\right)=\beta ; \quad \varepsilon_{k}(n, 0, \beta)=\varepsilon_{k}\left(n, k^{n}, \beta\right)=0 .
$$

\section{A Connection Between Optimal Cloud-Antichains and Pairs Extremal in the Meet}

In the Introduction we have defined the set $\mathcal{O}(n)$ of optimal cloud-antichains of length 2 in $[k]^{n}$. Now we define the set $\mathscr{E}(n)$ of pairs $(C, D)$ with $C, D \subset[k]^{n}$ which are extremal in the meet $C \wedge D$, that is,

$$
|C \wedge D|=\min \left\{\left|C^{\prime} \wedge D^{\prime}\right|: C^{\prime}, D^{\prime} \subset[k]^{n},\left|C^{\prime}\right|=|C|,\left|D^{\prime}\right|=|D|\right\} .
$$

\footnotetext{
1 For any $c=\left(c_{1}, \ldots, c_{n}\right) \in[k]^{n}$ we define a forward and a backward value by $\vec{v}(c)=$ $\sum_{i=1}^{n} k^{n-i} c_{i}$ and $\bar{v}(c)=\sum_{i=1}^{n} k^{i-1} c_{i}$, resp.. $\vec{v}$ and $\bar{v}$ evaluate elements as $k$-ary numbers when the components are written in natural order or in reversed order.
} 
Within $\mathcal{O}(n)$ there is the set $\mathscr{M}(n)$ of those pairs $(A, B)$ which are maximal in the sense that for all $(C, D) \in \mathscr{C} \mathscr{A} \mathscr{C}(n)$ with $|C|>|A|$ necessarily $|D|<|B|$.

Finally, we call $(A, B) \in \mathscr{C} \mathscr{A} \mathscr{C}(n) A$-saturated (resp. $B$-saturated), if there is no $\left(A, B^{\prime}\right) \in \mathscr{C} \mathscr{A} \mathscr{C}(n)$ with $B \subset B^{\prime}, B \neq B^{\prime}$ (resp. $\left(A^{\prime}, B\right) \in \mathscr{C} \mathscr{A} \mathscr{C}(n)$ with $\left.A \subset A^{\prime}, A \neq A^{\prime}\right)$ and we call $(A, B)$ saturated (or bisaturated), if it is $A$-saturated and $B$-saturated. We denote the set of all these saturated pairs by $\mathscr{S}(n)$.

Actually, fewer definitions would suffice for the proofs of our Theorems. However, clarity is gained by analysing in which generality properties used hold. We establish therefore first simple properties of the classes defined. It is immediately clear that

$$
\mathscr{M}(n)=\mathscr{O}(n) \cap \mathscr{S}(n)
$$

Our first result is of general nature.

\section{Lemma 1.}

(i) For $A, B \subset[k]^{n}$ we have $(A \backslash(A \wedge B), B \backslash(A \wedge B)) \in \mathscr{C} \mathscr{A} \mathscr{C}(n)$.

(ii) For $(A, B) \in \mathscr{C} \mathscr{A} \mathscr{C}(n)$ we have $A \cap(A \wedge B)=B \cap(A \wedge B)=A \cap(A \vee B)=$ $B \cap(A \vee B)=\varnothing$.

Proof. (i) Suppose that for $a \in A \backslash(A \wedge B)$ and $b \in B \backslash(A \wedge B)$ we have $a \geq b$, then $b=a \wedge b \in A \wedge B$, contradicting that $b \notin A \wedge B$ (the case $a \leq b$ is symmetrically the same).

(ii) If for instance $a \in A \cap(A \vee B)$, then a $b \in B$ exists with $b \leq a$. This contradicts the assumption $(A, B) \in \mathscr{C} \mathscr{A} \mathscr{C}(n)$.

For any $C \in[k]^{n}$ we define

$$
\varphi(C)=\left\{c \in[k]^{n}: c \notin C, \exists c^{\prime} \in C \text { with } c<c^{\prime}\right\} .
$$

Clearly, $C \cup \varphi(C)$ is an ideal ("downset").

With any pair $(A, B), A, B \subset[k]^{n}$, we associate a pair

$$
\left(A^{*}, B^{*}\right)=(A \cup \varphi(A), B \cup \varphi(B)) \text {. }
$$

Lemma 2. For $(A, B) \in \mathscr{S}(n)$ we have

$$
A^{*} \wedge B^{*}=A^{*} \cap B^{*}=\varphi(A)=\varphi(B) .
$$

Proof. If for instance $a \in \varphi(A)$ and $a \notin \varphi(B)$, then either $a \geq b$ for some $b \in B$ and for an $a^{\prime} \in A$ with $a^{\prime} \geq a$ the inequality $a^{\prime} \geq b$ contradicts $(A, B) \in \mathscr{C} \mathscr{A} \mathscr{C}(n)$, or $a$ is incomparable with all members of $B$, which contradicts the saturation of $(A, B)$. In any case we have $\varphi(A)=\varphi(B)$ and $A^{*} \wedge B^{*}=A^{*} \cap B^{*}$, because $A^{*}$ and $B^{*}$ are ideals. Finally, since $A \cap B=A \cap \varphi(A)=B \cap \varphi(B)=\varnothing$, we have

$$
A^{*} \cap B^{*}=\varphi(A)=\varphi(B) \text {. }
$$

Actually we need a related result. For any $\left(\alpha, f_{n}(\alpha)\right)$ there is a pair $(A, B) \in \mathcal{O}(n)$,

$$
(|A|,|B|)=\left(\alpha, f_{n}(\alpha)\right),
$$

with minimal total weight $\sum_{a \in A} W(a)$, where $W(a)=\sum_{t=1}^{n} a_{t}$. We denote the set of all such pairs by $\mathscr{O}_{*}(n)$. 
Lemma 3. For $(A, B) \in \mathcal{O}_{*}(n)(3.5)$ holds.

Proof. Since $(A, B)$ is $A$-saturated the previous argument gives $\varphi(B) \subset \varphi(A)$. For $a \in \varphi(A), a \notin \varphi(B)$ the case in which $a$ is incomparable with all members of $B$ is to be discussed. Here $A^{\prime}=(A \cup\{a\}) \backslash\left\{a^{\prime}\right\}, a^{\prime} \geq a$, has smaller total weight than $A$ and since $\left(A^{\prime}, B\right) \in \mathscr{C} \mathscr{A} \mathscr{C}(n)$ we get a contradiction. The remaining identities in (3.5) are established as previously.

Proposition 1. For $(A, B) \in \mathcal{O}_{*}(n)$ we have $\left(A^{*}, B^{*}\right) \in \mathscr{E}(n)$.

Proof. Set $\alpha^{*}=\left|A^{*}\right|$ and $\beta^{*}=\left|B^{*}\right|$. Now clearly

$$
\left|A^{*} \wedge B^{*}\right| \geq \mu_{k}\left(n, \alpha^{*}, \beta^{*}\right) \quad \text { (defined in Section 2) }
$$

and suppose that here strict inequality holds. By Theorem $2[5]$ there are ideals $A_{1}, B_{1}$ with cardinalities $\left|A_{1}\right|=\alpha^{*},\left|B_{1}\right|=\beta^{*}$ and $\left|A_{1} \wedge B_{1}\right|=\mu_{k}\left(n, \alpha^{*}, \beta^{*}\right)$. Therefore

$$
\begin{aligned}
\left|A_{1} \backslash\left(A_{1} \wedge B_{1}\right)\right| & =\alpha^{*}-\mu\left(n, \alpha^{*}, \beta^{*}\right)>\alpha^{*}-\left|A^{*} \wedge B^{*}\right| \\
& =\left|A^{*}\right|-|\varphi(A)| \text { by Lemma } 3 .
\end{aligned}
$$

By definition of $A^{*}\left|A^{*}\right|-|\varphi(A)|=|A|$ and thus $\left|A_{1} \backslash\left(A_{1} \wedge B_{1}\right)\right|>|A|$. Symmetrically $\left|B_{1} \backslash\left(A_{1} \wedge B_{1}\right)\right|>|B|$.

Since $\left(A_{1} \backslash\left(A_{1} \wedge B_{1}\right), B_{1} \backslash\left(A_{1} \wedge B_{1}\right)\right) \in \mathscr{C} \mathscr{A} \mathscr{C}(n)$ (by (i) in Lemma 1) we obtain a contradiction to the optimality of $(A, B)$.

\section{A Formula for $f_{n}$ via $\mathscr{E}(n)$}

Let us define for $0 \leq \gamma \leq k^{n}$

$$
M_{\gamma}=\left\{(\alpha, \beta): 0 \leq \alpha, \beta \leq k^{n}, \alpha-\mu_{k}(n, \alpha, \beta)=\gamma\right\} .
$$

Here all numbers are non-negative integers. Note that $M_{\gamma} \neq \varnothing$, because $(\gamma, 0) \in M_{\gamma}$.

Lemma 4. For $0 \leq \gamma \leq k^{n}$

$$
f_{n}(\gamma)=\max _{(\alpha, \beta) \in M_{\gamma}}\left(\beta-\mu_{k}(n, \alpha, \beta)\right)
$$

and the maximum can be achieved for a pair of ideals.

Proof. Let $(A, B) \in O_{*}(n)$ satisfy $|A|=\gamma,|B|=f_{n}(\gamma)$. By Proposition $1\left(\left|A^{*}\right|,\left|B^{*}\right|\right)=$ $\left(\gamma+\mu_{k}\left(n,\left|A^{*}\right|,\left|B^{*}\right|\right), f_{n}(\gamma)+\mu_{k}\left(n,\left|A^{*}\right|,\left|B^{*}\right|\right)\right)$ and hence $\left(\left|A^{*}\right|,\left|B^{*}\right|\right) \in M_{\gamma}$. Therefore,

$$
f_{n}(\gamma)=\left|B^{*}\right|-\mu_{k}\left(n,\left|A^{*}\right|,\left|B^{*}\right|\right) \leq \max _{(\alpha, \beta) \in M_{\gamma}}\left(\beta-\mu_{k}(n, \alpha, \beta)\right) .
$$

To establish the reverse inequality, suppose the maximum is assumed at $\left(\alpha^{\prime}, \beta^{\prime}\right) \in M_{y}$ and let $\left(A^{\prime}, B^{\prime}\right)$ satisfy $\left|A^{\prime}\right|=\alpha^{\prime},\left|B^{\prime}\right|=\beta^{\prime}$ and $\left|A^{\prime} \wedge B^{\prime}\right|=\mu_{k}\left(n, \alpha^{\prime}, \beta^{\prime}\right)$. By Theorem 2 [5] $A^{\prime}$ and $B^{\prime}$ can be assumed to be ideals. Then $A=A^{\prime} \backslash\left(A^{\prime} \wedge B^{\prime}\right)$ and 
$B=B^{\prime} \backslash\left(A^{\prime} \wedge B^{\prime}\right)$ satisfy $(A, B) \in \mathscr{C} \mathscr{A} \mathscr{C}(n)$ (by Lemma 1$),|A|=\alpha^{\prime}-\mu_{k}\left(n, \alpha^{\prime}, \beta^{\prime}\right)=$ $\gamma$, and $|B|=\beta^{\prime}-\mu_{k}\left(n, \alpha^{\prime}, \beta^{\prime}\right) \leq f_{n}(\gamma)$. Therefore

$$
f_{n}(\gamma)=\left|B^{*}\right|-\mu_{k}\left(n,\left|A^{*}\right|,\left|B^{*}\right|\right)=\max _{(\alpha, \beta) \in M_{\gamma}}\left(\beta-\mu_{k}(n, \alpha, \beta)\right) .
$$

Since $A^{*}$ and $B^{*}$ are ideals, the maximum in (4.2) at $\left(\alpha^{\prime}, \beta^{\prime}\right)$ is assumed for a pair of ideals. Actually, with the aid of Theorem 4 [5] a stronger conclusion can be drawn

Lemma 5. If the maximum in (4.2) is assumed at $\left(\alpha^{*}, \beta^{*}\right)$, then it can be attained by letting $A^{*}$ be the $\alpha^{*}$ smallest and written backwards, $B^{*}$ be the $\beta^{*}$ smallest $n$-digit $k$-ary numbers.

Next we establish a relation between $\gamma$ and a pair $\left(\alpha^{*}, \beta^{*}\right)$ at which the maximum occurs.

Lemma 6. If the maximum in (4.2) is assumed at $\left(\alpha^{*}, \beta^{*}\right)$ and if $\gamma \leq k^{n-2}$, then $\alpha^{*} \leq k^{n-1}$ and $\beta^{*} \geq(k-1) k^{n-1}$.

Proof. This is the first argument, which not just generalizes that of [9]. However, it starts as previously. Let us consider the pair $\left(k \gamma,(k-1) k^{n-1}\right)$ and derive with (i) in Theorem 5 [5] (for $p=k-2)$ and $\mu_{k}(n, \alpha, \beta)=\mu_{k}(n, \beta, \alpha)$ that $\left(k \gamma,(k-1) k^{n-1}\right) \in$ $M_{\gamma}$, because

$$
\begin{aligned}
k \gamma-\mu_{k}\left(n, k \gamma,(k-1) k^{n-1}\right) & =k \gamma-\mu_{k}\left(n-1, \gamma, k^{n-1}\right)-(k-2) \gamma \\
& =k \gamma-\gamma-(k-2) \gamma=\gamma .
\end{aligned}
$$

Therefore

$$
\begin{aligned}
& \max _{(\alpha, \beta) \in M_{\gamma}}\left(\beta-\mu_{k}(n, \alpha, \beta)\right) \geq(k-1) k^{n-1}-(k-1) \gamma \\
& \quad=(k-1) k^{n-1}-(k-1)\left(\alpha^{\prime}-\mu_{k}\left(n, \alpha^{\prime}, \beta^{\prime}\right)\right) \quad \text { for all }\left(\alpha^{\prime}, \beta^{\prime}\right) \in M_{\gamma} .
\end{aligned}
$$

In particular we get at a maximum $\left(\alpha^{*}, \beta^{*}\right)$

$$
\beta^{*}-\mu_{k}\left(n, \alpha^{*}, \beta^{*}\right) \geq(k-1) k^{n-1}-(k-1)\left(\alpha^{*}-\mu_{k}\left(n, \alpha^{*}, \beta^{*}\right)\right)
$$

or equivalently

$$
\beta^{*}+(k-1) \alpha^{*}-k \mu_{k}\left(n, \alpha^{*}, \beta^{*}\right) \geq(k-1) k^{n-1} .
$$

Since for any $C, D \subset[k]^{n}|C||D| \leq|C \wedge D||C \vee D| \leq|C \wedge D| k^{n}$, we also have

$$
\mu_{k}\left(n, \alpha^{*}, \beta^{*}\right) \geq \frac{\alpha^{*} \beta^{*}}{k^{n}} .
$$

Combination of (4.3) and (4.4) yields

$$
\beta^{*}+(k-1) \alpha^{*}-\frac{\alpha^{*} \beta^{*}}{k^{n-1}} \geq(k-1) k^{n-1} .
$$


Write now $\beta^{*}=(k-1) k^{n-1}+\delta, \alpha^{*}=k^{n-1}+\varepsilon$ and verify that

$$
\begin{aligned}
\beta^{*}+(k-1) \alpha^{*}-\frac{\alpha^{*} \beta^{*}}{k^{n-1}=} & (k-1) k^{n-1}+\delta+(k-1) k^{n-1} \\
& +(k-1) \varepsilon-\frac{\left(k^{n-1}+\varepsilon\right)\left((k-1) k^{n-1}+\delta\right)}{k^{n-1}} \\
= & (k-1) k^{n-1}-\frac{\varepsilon \delta}{k^{n-1}} .
\end{aligned}
$$

We conclude from (4.5) that $\varepsilon \delta \leq 0$. In view of (4.2) $\beta^{*} \geq \alpha^{*}$, but if $k-1>1$ this does not imply (as in the case $k=2$ ) that $\delta \geq 0$ and $\varepsilon \leq 0$.

Suppose, for contradiction, that either $\delta \leq 0$ and $\varepsilon>0$, or $\delta<0$ and $\varepsilon=0$. By definition of $\left(\alpha^{*}, \beta^{*}\right), f_{n}(\gamma)=\beta^{*}-\mu_{k}\left(n, \alpha^{*}, \beta^{*}\right)$ and $\left(\alpha^{*}, \beta^{*}\right) \in M_{\gamma}$, so $\alpha^{*}-\mu_{k}\left(n, \alpha^{*}, \beta^{*}\right)=\gamma$. Thus $f_{n}(\gamma)-\gamma=\beta^{*}-\alpha^{*}$. From the preceding verification it follows that $\beta^{*}-\alpha^{*}<(k-1) k^{n-1}-k^{n-1}=(k-2) k^{n-1}$. Thus

$$
f_{n}(\gamma)-\gamma<(k-2) k^{n-1} \text {. }
$$

Next observe that the pair

$$
(A, B)=\left(\{0\} \times\{k-1\} \times \prod_{i=3}^{n}[k],\{1, \ldots, k-1\} \times\{0, \ldots, k-2\} \times \prod_{t=3}^{n}[k]\right)
$$

is a cloud antichain with $|A|=k^{n-2}$ and $|B|=(k-1)^{2} k^{n-2}$, so

$$
f_{n}\left(k^{n-2}\right) \geq(k-1)^{2} k^{n-2} .
$$

It follows from (4.6) and (4.7) that

$$
f_{n}\left(k^{n-2}\right)-k^{n-2} \geq(k-1)^{2} k^{n-2}-k^{n-2}=(k-2) k^{n-1}>f_{n}(\gamma)-\gamma .
$$

But $f_{n}(\cdot)$ is a decreasing function and $\gamma \leq k^{n-2}$ by hypothesis so we have the contradiction

$$
f_{n}\left(k^{n-2}\right)-k^{n-2}>f_{n}(\gamma)-\gamma \geq f_{n}\left(k^{n-2}\right)-k^{n-2}
$$

\section{Proof of Theorem 1}

Starting with $(A, B) \in \mathcal{O}_{*}(n),|A|=\gamma,|B|=f_{n}(\gamma)$ we know from Proposition 1 that $\left(A^{*}, B^{*}\right) \in \mathscr{E}(n)$ and that $\alpha^{*}=\left|A^{*}\right|=\gamma+\mu_{k}\left(n, \alpha^{*}, \beta^{*}\right), \beta^{*}=\left|B^{*}\right|=f_{n}(\gamma)+$ $\mu_{k}\left(n, \alpha^{*}, \beta^{*}\right)$. The same parameter values can be obtained with $A^{*}, B^{*}$ being ideals and having the numberings of Lemma 5. Then the sequences in $A=A^{*} \backslash\left(A^{*} \wedge B^{*}\right)$ are all zero in the last component, because $\left|A^{*}\right|=\alpha^{*} \leq k^{n-1}$ by Lemma 6 , and they are all $k-1$ in the first component, because $\left|B^{*}\right| \geq(k-1) k^{n-1}$ again by Lemma 6 and thus all elements of $A^{*}$ beginning with an element of $\{0,1, \ldots, k-2\}$ are also in $A^{*} \wedge B^{*}$ and thus not in $A$. This means that only elements of $A^{*}$ beginning with a $k-1$ can be members of $A$. 


\section{Proof of Theorem 2}

We make use of the notation

$$
\begin{aligned}
& \varphi(D)=\left\{d \in[k]^{n}: d \notin D, \exists d^{\prime} \in D \text { with } d<d^{\prime}\right\}, \\
& \Psi(D)=\left\{d \in[k]^{n}: d \notin D, \exists d^{\prime} \in D \text { with } d>d^{\prime}\right\},
\end{aligned}
$$

and

$$
\sigma(D)=\left\{c \in[k]^{n}: c \Varangle d, c \nsucceq d \text { for all } d \in D\right\} .
$$

Let now $(A, B) \in \mathcal{O}_{*}(n)$ have an $A$ structured as in Theorem 1: $a=\left(a_{1}, a_{2}, \ldots, a_{n}\right) \in$ $A$ implies $a_{1}=k-1, a_{n}=0$. Necessarily $B=\sigma(A)$ and it can be described as a union of four disjoint sets:

Imagine $b \in[k]^{n}$ to be written in the form $\left(b_{1}, \ldots, b_{n}\right)$ and set

$$
\begin{aligned}
B_{1}= & \left\{b \in[k]^{n}: b_{1} \in\{0, \ldots, k-2\}, b_{n} \in\{1, \ldots, k-1\},\left(b_{2}, \ldots, b_{n-1}\right) \in[k]^{n-2}\right\}, \\
B_{2}= & \left\{b \in[k]^{n}: b_{1} \in\{0, \ldots, k-2\}, b_{n}=0,\left(b_{2}, \ldots, b_{n-1}\right) \in \Psi\left(A_{0}\right) \cup \sigma\left(A_{0}\right)\right\}, \\
& \text { where } A_{0}=\left\{\left(a_{2}, \ldots, a_{n-1}\right) \in[k]^{n-2}:\left(k-1, a_{2}, \ldots, a_{n-1}, 0\right) \in A\right\}, \\
B_{3}= & \left\{b \in[k]^{n}: b_{1}=k-1, b_{n} \in\{1, \ldots, k-1\},\left(b_{2}, \ldots, b_{n-1}\right) \in \varphi\left(A_{0}\right) \cup \sigma\left(A_{0}\right)\right\}, \\
B_{4}= & \left\{b \in[k]^{n}: b_{1}=k-1, b_{n}=0,\left(b_{2}, \ldots, b_{n-1}\right) \in \sigma\left(A_{0}\right)\right\} .
\end{aligned}
$$

Then

$$
\begin{aligned}
|B|= & \left|B_{1} \cup B_{2} \cup B_{3} \cup B_{4}\right|=(k-1)^{2} k^{n-2}+(k-1)\left(\left|\Psi\left(A_{0}\right)\right|+\left|\varphi\left(A_{0}\right)\right|\right) \\
& +(2 k-1)\left|\sigma\left(A_{0}\right)\right|,
\end{aligned}
$$

and since

$$
\left|\Psi\left(A_{0}\right)\right|+\left|\varphi\left(A_{0}\right)\right|+\left|\sigma\left(A_{0}\right)\right|=k^{n-2}-\left|A_{0}\right|=k^{n-2}-\gamma,
$$

we conclude that

$$
\begin{aligned}
f_{n}(\gamma) & =|B|=(k-1)^{2} k^{n-2}+(k-1)\left(k^{n-2}-\gamma\right)+k\left|\sigma\left(A_{0}\right)\right| \\
& \leq(k-1)^{2} k^{n-2}+(k-1)\left(k^{n-2}-\gamma\right)+k f_{n-2}(\gamma) \\
& =(k-1) k^{n-1}+k f_{n-2}(\gamma)-(k-1) \gamma .
\end{aligned}
$$

Conversely, if we choose $A_{0} \subset[k]^{n-2},\left|A_{0}\right|=\gamma$ such that $\left(A_{0}, \sigma\left(A_{0}\right)\right) \in$ $\mathcal{O}_{*}(n-2)$, then $\left|\sigma\left(A_{0}\right)\right|=f_{n-2}(\gamma)$ and constructing $A$ and $B$ as before, we obtain

$$
|B|=(k-1) k^{n-1}+k f_{n-2}(\gamma)-(k-1) \gamma .
$$

Writing (i) in the form

$$
f_{n+2}(\gamma)=(k-1)\left(k^{n+1}-\gamma\right)+k f_{n}(\gamma)
$$

(ii) follows by induction on $s$. 


\section{A Corollary}

Since there exists $(A, B) \in \mathscr{C} \mathscr{A} \mathscr{C}(n)$ with $|A|=\gamma$ and $|B|=f_{n}(\gamma)$, it is immediate that

$$
f_{n}\left(f_{n}(\gamma)\right) \geq \gamma
$$

Since the function $f_{n}$ is decreasing we have therefore

$$
f_{n}\left(f_{n}\left(f_{n}(\gamma)\right)\right) \leq f_{n}(\gamma)
$$

On the other hand there exists $\left(A^{\prime}, B^{\prime}\right) \in \mathscr{C} \mathscr{A} \mathscr{C}(n)$ with $\left|A^{\prime}\right|=f_{n}\left(f_{n}(\gamma)\right)$ and $\left|B^{\prime}\right|=$ $f_{n}(\gamma)$. Hence

$$
f_{n}\left(f_{n}\left(f_{n}(\gamma)\right)\right) \geq f_{n}(\gamma)
$$

and from (7.2), (7.3) we conclude

$$
f_{n}\left(f_{n}\left(f_{n}(\gamma)\right)\right)=f_{n}(\gamma) \quad \text { for all } \quad \gamma \leq k^{n} .
$$

For $\gamma+1 \leq k^{n-2}$ the recursion (i) in Theorem 2 applies and, since $f_{n-2}(\gamma+1) \leq$ $f_{n-2}(\gamma)$, we have $f_{n}(\gamma+1)=(k-1) k^{n-1}+k f_{n-2}(\gamma+1)-(k-1)(\gamma+1) \leq$ $(k-1) k^{n-1}+k f_{n-2}(\gamma)-(k-1) \gamma-(k-1)=f_{n}(\gamma)-(k-1)$, and hence

$$
f_{n}(\gamma+1)<f_{n}(\gamma) \quad \text { for all } 0 \leq \gamma<k^{n-2} \text {. }
$$

Suppose for some $\gamma, 0 \leq \gamma<k^{n-2}$, strict inequality in (7.1) holds, i.e.

$$
f_{n}\left(f_{n}(\gamma)\right)=\gamma_{1} \geq \gamma+1
$$

then, applying (7.4), we have

$$
f_{n}\left(f_{n}\left(f_{n}(\gamma)\right)\right)=f_{n}\left(\gamma_{1}\right)=f_{n}(\gamma)
$$

which contradicts (7.5).

We summarize these findings

\section{Corollary.}

(i) $f_{n}\left(f_{n}(\gamma)\right) \geq \gamma$ for all $\gamma \leq k^{n}$

(ii) $f_{n}\left(f_{n}(\gamma)\right)=\gamma$ for $0 \leq \gamma<k^{n-2}$.

(iii) $f_{n}\left(f_{n}\left(f_{n}(\gamma)\right)\right)=f_{n}(\gamma)$ for all $\gamma \leq k^{n}$.

\section{On Problem I: an Equality Characterization}

What can we say about $(A, B) \in \mathscr{C} \mathscr{A} \mathscr{C}_{k}(n)$ for which

$$
\sqrt{k^{n}}=\sqrt{|A|}+\sqrt{|B|}
$$

holds?

First we derive an auxiliary result. 
Lemma 7. For an $(A, B) \in \mathscr{C} \mathscr{A} \mathscr{C}_{k}$ (n) satisfying (8.1) necessarily we have

(i) $A$ and $B$ are convex.

(ii) $(A, B) \in \mathcal{O}(n)$.

(iii) $(A, B)$ is bisaturated.

(iv) $\varphi(A)=\varphi(B)=A \wedge B$ and (symmetrically) $\Psi(A)=\Psi(B)=A \vee B$.

Proof. Statements (i), (ii) and (iii) are trivially true, because otherwise we could increase $\sqrt{|A|}+\sqrt{|B|}$.

Lemma 2 implies

$$
\varphi(A)=\varphi(B)=A^{*} \wedge B^{*} \supset A \wedge B .
$$

Also we have by the disjointness properties and the inclusion $\Psi(A) \supset A \vee B$

$$
\begin{aligned}
k^{n} & \geq|A|+|B|+|\varphi(A)|+|\Psi(A)| \geq|A|+|B|+|A \wedge B|+|A \vee B| \\
& \left.\geq(\sqrt{|A|}+\sqrt{|B|})^{2} \quad \text { by }(1.7)\right)
\end{aligned}
$$

and (8.1) yields

$$
|\varphi(A)|+|\Psi(A)|=|A \wedge B|+|A \vee B| .
$$

Thus (8.2) gives finally (iv).

Lemma 8. If (8.1) holds, then

(i) $\alpha+\beta=k^{n}$ and $\sqrt{\alpha-\mu_{k}(n, \alpha, \beta)}+\sqrt{\beta-\mu_{k}(n, \alpha, \beta)}=\sqrt{k^{n}}$

or

(ii) $\alpha+\beta=k^{n} \quad$ and $\quad \mu_{k}(n, \alpha, \beta)=\frac{\alpha \beta}{k^{n}}$.

Proof. By Lemma 4 we know that for $(A, B) \in \mathcal{O}(n)$ we can assume that

$$
|A|=\alpha-\mu_{k}(n, \alpha, \beta), \quad|B|=\beta-\mu_{k}(n, \alpha, \beta)
$$

and there are ideals $A^{*}, B^{*}$ with $\left|A^{*}\right|=\alpha,\left|B^{*}\right|=\beta,\left|A^{*} \wedge B^{*}\right|=\mu_{k}(n, \alpha, \beta)$ $\left(0 \leq \alpha, \beta \leq k^{n}\right)$. Since (8.1) holds, by (iii) in Lemma $7(A, B) \in S(n)$ and hence by Lemma $2 \mu_{k}(n, \alpha, \beta)=\left|A^{*} \wedge B^{*}\right|=|\varphi(A)|$. Now by (iv) in Lemma 7 we conclude that

$$
\mu_{k}(n, \alpha, \beta)=|\varphi(A)|=|A \wedge B| .
$$

Also $|A \vee B|=\mu_{k}(n, \alpha, \beta)$, because $|A \wedge B|+|A \vee B|=2 \sqrt{|A \wedge B||A \vee B|}$, iff $|A \wedge B|=|A \vee B|$.

Recall also that under (8.1) $k^{n}=|A|+|B|+|A \wedge B|+|A \vee B|$ and by (8.3) we have

$$
|A|+|A \wedge B|=\alpha, \quad|B|+|A \vee B|=\beta .
$$

Theorem 3. $\sqrt{|A|}+\sqrt{|B|}=\sqrt{k^{n}}$ holds for $(A, B) \in \mathscr{C A A C} \mathscr{C}_{k}(n)$ if and only if, for some integer $l, 0 \leq l \leq k^{m}$ 


$$
\text { (i) } n=2 m, \quad|A|=l^{2}, \quad|B|=\left(k^{m}-l\right)^{2}
$$

or

$$
\text { (ii) } n=2 m+1, \quad|A|=k l^{2}, \quad|B|=k\left(k^{m}-l\right)^{2} .
$$

Proof. Sufficiency of (i) or (ii) follows by a construction, which generalizes that of Section 8 in [9] from $k=2$ to general $k$. In case (i) it goes as follows. Set

Let $\underline{A}_{m}(s)$ (resp. $\left.\bar{A}_{m}(s)\right)$ be the $s$ smallest (resp. largest) weight elements of $[k]^{m}$.

$$
\begin{aligned}
& A=\left\{a \in[k]^{2 m}: a=\left(a_{1}, a_{2}\right), a_{1} \in \underline{A}_{m}(l), a_{2} \in \bar{A}_{m}(l)\right\}, \\
& B=\left\{b \in[k]^{2 m}: b=\left(b_{1}, b_{2}\right), b_{1} \in \bar{A}_{m}\left(k^{m}-l\right), b_{2} \in \underline{A}_{m}\left(k^{m}-l\right)\right\},
\end{aligned}
$$

and verify that $(A, B) \in \mathscr{C} \mathscr{A} \mathscr{C}(n),|A|=l^{2}$, and $|B|=\left(k^{m}-l\right)^{2}$. In case (ii) choose $\left(A^{\prime}, B^{\prime}\right)=(A \times[k], B \times[k])$.

To see the necessity we use the following result, which is a special case of our forthcoming Theorem 2 in [10], which corrects Theorem 6 of $[5]: \varepsilon_{k}(n, \alpha, \beta)=$ $\mu_{k}(n, \alpha, \beta)-\frac{\alpha \beta}{k^{n}}=0$ iff (i) $k^{i}\left|\alpha, k^{i+1} \nmid \alpha \Rightarrow k^{n-i}\right| \beta$ or (ii) $\alpha$ or $\beta$ equals $k^{n}$ or 0 .

In our case $\mu_{k}(n, \alpha, \beta)=\mu_{k}\left(n, \alpha, k^{n}-\alpha\right)=\frac{\alpha\left(k^{n}-\alpha\right)}{k^{n}}=\alpha-\frac{\alpha^{2}}{k^{n}}$ is possible only when $k^{n} \mid \alpha^{2}$ and we can use the relations

$$
k^{i}\left|\alpha, \quad k^{i+1} \nmid \alpha \Rightarrow k^{i}\right| \beta, \quad \beta=k^{n}-\alpha .
$$

However, we have also $k^{i}\left|\alpha, k^{i+1}\right| \alpha \Rightarrow k^{n-i} \mid k^{n}-\alpha$ and $k^{i+1} \nmid k^{n}-\alpha$ and therefore necessarily $i \geq n-i$. Hence (ii) in Lemma 8 holds iff $k^{i} \mid \alpha$ where

1.) $i \geq m$, if $n=2 m$

2.) $i \geq m+1$, if $n=2 m+1$.

Now we have $|A|=\alpha-\mu_{k}(n, \alpha, \beta)=\frac{\alpha^{2}}{k^{2}}$ and

1.) $n=2 m, \alpha=k^{m} \cdot l(l$ integral $),|A|=l^{2},|B|=\left(k^{m}-l\right)^{2}$

2.) $n=2 m+1, \alpha=k^{m+1} l,|A|=k l^{2},|B|=k\left(k^{m}-l\right)^{2}$.

\section{An Asymptotic Result}

The recursive formula of Theorem 2 in conjunction with Theorem 3 allow to estimate the growth of $f_{n}$.

\section{Theorem 4.}

(i) For any $\gamma \in \mathbb{N}$ (the natural numbers) such that $\sqrt{\gamma}+\sqrt{f_{n}(\gamma)}<\sqrt{k^{n}}$

$$
\lim _{s \rightarrow \infty}\left(\sqrt{k^{n+2 s}}-\sqrt{\gamma}\right)^{2}-f_{n+2 s}(\gamma)=\infty
$$


(ii) For any $\gamma \in \mathbb{N}$

$$
\lim _{n \rightarrow \infty} \frac{\left(\sqrt{k^{n}}-\sqrt{\gamma}\right)^{2}}{f_{n}(\gamma)}=1
$$

Proof. (i) We choose $n$ such that $\gamma \leq k^{n}$ and let $\varepsilon_{n}(\gamma)=\left(\sqrt{k^{n}}-\sqrt{\gamma}\right)^{2}-f_{n}(\gamma)>0$. The recursion (ii) in Theorem 2 can be written in the form

$$
f_{n+2 s}(\gamma)=\left(\sqrt{k^{n+2 s}}-\sqrt{\gamma}\right)^{2}-k^{s}\left(\left(\sqrt{k^{n}}-\sqrt{\gamma}\right)^{2}-f_{n}(\gamma)\right)
$$

which implies

$$
\left(\sqrt{k^{n+2 s}}-\sqrt{\gamma}\right)^{2}-f_{n+2 s}(\gamma)=k^{s} \varepsilon_{n}(\gamma)
$$

and $k^{s} \varepsilon_{n}(\gamma) \rightarrow \infty(s \rightarrow \infty)$.

(ii) For any $\gamma$ and $n$ so large that $\gamma<k^{n}$ we write

$$
\frac{\left(\sqrt{k^{n+2 s}}-\sqrt{\gamma}\right)^{2}}{f_{n+2 s}(\gamma)}=\frac{\left(\sqrt{k^{n+2 s}}-\sqrt{\gamma}\right)^{2}}{\left(k^{s}-1\right)\left(k^{n+s}-\gamma\right)+k^{s} f_{n}(\gamma)}
$$

and notice that

$$
\lim _{s \rightarrow \infty} \frac{\left(\sqrt{k^{n+2 s}}-\sqrt{\gamma}\right)^{2}}{\left(k^{s}-1\right)\left(k^{n+s}-\gamma\right)+k^{s} f_{n}(\gamma)}=1
$$

\section{A Divisibility Property of Optimal and Bisaturated Pairs}

We present here a result, which we later use and which is interesting in itself.

Theorem 5. Let $(A, B)$ be optimal and bisaturated, that is, $(A, B) \in \mathcal{O}(n) \cap \mathscr{S}(n)=\mathscr{M}(n)$. Then a maximum pair $\left(\alpha^{*}, \beta^{*}\right)$ with $\alpha^{*}-\mu_{k}\left(n, \alpha^{*}, \beta^{*}\right)=|A|, \beta^{*}-\mu_{k}\left(n, \alpha^{*}, \beta^{*}\right)=|B|$ has the properties $k \mid \alpha$ and $k \mid \beta$. In particular

$$
k \mid(|A|-|B|)
$$

Proof. Since by definition of optimality for a $\gamma|A|=\gamma$ and $|B|=f_{n}(\gamma)$ we know from Lemma 4 that

$$
f_{n}(\gamma)=\max _{(\alpha, \beta) \in M_{\gamma}}\left(\beta-\mu_{k}(n, \alpha, \beta)\right),
$$

where

$$
M_{\gamma}=\left\{(\alpha, \beta): 0 \leq \alpha, \beta \leq k^{n}, \alpha-\mu_{k}(n, \alpha, \beta)=\gamma\right\} .
$$

We also know from Lemma 5 that at a maximum assuming pair $\left(\alpha^{*}, \beta^{*}\right)$ there is a realisation $(A, B)=\left(A^{*} \backslash\left(A^{*} \wedge B^{*}\right), B^{*} \backslash\left(A^{*} \wedge B^{*}\right)\right)$, where $A^{*}$ are the $\alpha^{*}$ smallest and written backwards, $B^{*}$ are the $\beta^{*}$ smallest $n$-digit $k$-ary numbers.

First we show that integer $\alpha^{*}-1$ represented lexicographically backwards does not appear in the list of $B^{*}$. Namely otherwise we have

$$
\mu_{k}\left(n, \alpha^{*}-1, \beta\right)=\mu_{k}\left(n, \alpha^{*}, \beta^{*}\right)-1
$$


and thus

$$
\alpha^{*}-1-\mu_{k}\left(n, \alpha^{*}-1, \beta\right)=\alpha^{*}-1-\left(\mu_{k}\left(n, \alpha^{*}, \beta^{*}\right)-1\right)=\gamma .
$$

Hence $\left(\alpha^{*}-1, \beta^{*}\right) \in M_{\gamma}$. But we then have the contradiction

$$
\beta^{*}-\mu_{k}\left(n, \alpha^{*}-1, \beta\right)=f_{n}(\gamma)+1>f_{n}(\gamma)=\max _{(\alpha, \beta) \in M_{y}}\left(\beta-\mu_{k}(n, \alpha, \beta)\right) .
$$

Also $\beta^{*}-1$ written lexicographically forward does not appear in $A^{*}$, because otherwise $\mu_{k}\left(n, \alpha^{*}, \beta^{*}-1\right)=\mu_{k}\left(n, \alpha^{*}, \beta^{*}\right)-1$ and thus

$$
\beta^{*}-1-\mu_{k}\left(n, \alpha^{*}, \beta^{*}-1\right)=f_{n}(\gamma), \alpha^{*}-\mu_{k}\left(n, \alpha^{*}, \beta^{*}-1\right)=\gamma+1
$$

in contradiction to bisaturation.

We interpret now these properties in terms of the structures of $A^{*}$ and $B^{*}$ under the assumption $k \nmid \beta^{*}$ and derive a contradiction. By our supposition $k \nmid \beta^{*}$ for the last digit $d_{n}\left(\beta^{*}-1\right)$ (i.e. the $n$-th component of $\left.\beta^{*}-1\right)$ of integer $\beta^{*}-1$ (the biggest number in $B^{*}$ ) we have

$$
d_{n}\left(\beta^{*}-1\right) \neq k-1
$$

Let integer $\beta^{*}-1$ be the $l$-th lexicographic element, when it is read backwards (as the numbers in $A^{*}$ ). We just proved that it does not appear in $A^{*}$ and so $l \geq \alpha^{*}$. Since $d_{n}\left(\beta^{*}-1\right) \neq k-1$, we get $d_{n}\left(\beta^{*}\right)=d_{n}\left(\beta^{*}-1\right)+1$ and so integer $\beta^{*}$ is the $\left(l+k^{n-1}\right)$-th element when read lexicographically backwards. Therefore integer $\beta^{*}$ is also not in the list $A^{*}$. This means that $\mu_{r}\left(n, \alpha^{*}, \beta^{*}+1\right)=\mu_{k}\left(n, \alpha^{*}, \beta^{*}\right)$ and thus

$$
\alpha^{*}-\mu_{k}\left(n, \alpha^{*}, \beta^{*}+1\right)=\gamma, \beta^{*}+1-\mu_{k}\left(n, \alpha^{*}, \beta^{*}+1\right)=f_{n}(\gamma)+1,
$$

which contradicts optimality and completes the proof.

Remark. The relation $k \mid(|A|-|B|)$ need not hold, if we require only $(A, B) \in \mathcal{O}(n)$ or only $(A, B) \in \mathscr{S}(n)$.

Example 1. $n=4, k=2, \gamma=8, f_{4}(8)=1$ because $f_{4}(8)<2$ by $(1.7)$ and $f_{4}(8) \geq$ $f_{4}(9)=1$ by Theorem 3 (i), but $2 \nmid 8-1$.

Example 2. $n=6, k=2,(A, B) \in \mathscr{S}(6)$ (by inspection), $|A|=3,|B|=20,2 \nmid(20-3)$.

$$
\begin{aligned}
A= & \{111100,110011,001111\}, \quad B=B_{1} \cup B_{2}, \\
B_{1}= & \{101010,101001,100110,100101,011010, \\
& 011001,010110,010101,111010,111001\}, \\
B_{2}= & \{110110,110101,101110,101101,011110, \\
& 011101,101011,100111,011011,010111\} .
\end{aligned}
$$

Note also that here $\varphi(A)=\varphi(B) \neq A \wedge B$, because $(000000) \notin A \wedge B$. 


\section{Solution of Problem III}

It suffices to study the set

$$
Z(G(n))=\left\{(A, B) \in \mathscr{C} \mathscr{A} \mathscr{C}_{k}(n):|A|=G(n) \leq|B|\right\},
$$

where $G(n)$ is defined in the Introduction.

Lemma 9. The set $Z(G(n))$ consists only of pairs $(A, B)$ for which $|A|=|B|=G(n)$ or equivalently $f_{n}(G(n))=G(n)$.

Proof. Clearly there are $(A, B) \in Z(G)$ which are optimal and bisaturated. It suffices to show that for such a pair $|A|=|B|$.

We know from Lemma 5 that there exist integers $\alpha^{*}, \beta^{*}, 0 \leq \alpha^{*}, \beta^{*} \leq k^{n}$ such that

$$
\begin{aligned}
& \alpha^{*}-\mu_{k}\left(n, \alpha^{*}, \beta^{*}\right)=|A|=G, \\
& \beta^{*}-\mu_{k}\left(n, \alpha^{*}, \beta^{*}\right)=|B|=f_{n}(|A|),
\end{aligned}
$$

where $A$ and $B$ are the not intersected parts of ideals $\overleftarrow{A^{*}}$ and $\overrightarrow{B^{*}}$ with $\left|\overleftarrow{A^{*}}\right|=\alpha^{*}$, $\left|\overrightarrow{B^{*}}\right|=\beta^{*}$. Here $\overleftarrow{A^{*}}$ are the $\alpha^{*}$ smallest and written backwards, $\overrightarrow{B^{*}}$ are the $\beta^{*}$ smallest $n$-digit $k$-ary numbers.

Then $|B|>|A|$ would mean $\beta^{*}>\alpha^{*}$ and from Theorem $5 \beta^{*} \geq \alpha^{*}+k$. At first we claim that

$$
\mu_{k}\left(n, \alpha^{*}, \alpha^{*}\right)=\mu_{k}\left(n, \alpha^{*}, \beta^{*}\right) .
$$

Otherwise, since $\mu_{k}(n, \alpha, \beta)$ is an increasing function of $\beta$, we'd have $\mu_{\mathrm{k}}\left(n, \alpha^{*}, \alpha^{*}\right)<\mu_{\mathrm{k}}\left(n, \alpha^{*}, \beta^{*}\right)$. Then if $\overleftarrow{A^{*}}$ and $\overrightarrow{A^{*}}$ denote respectively the first written backward and the first written forward $\alpha^{*}$ elements of $[k]^{n}$, and $A^{\prime}=\overleftarrow{A^{*}} \backslash \overleftarrow{A^{*}} \wedge \overrightarrow{A^{*}}$, $B^{\prime}=\overrightarrow{A^{*}} \backslash \vec{A}^{*} \wedge \overrightarrow{A^{*}}$, then $\left(A^{\prime}, B^{\prime}\right) \in \mathscr{C} \mathscr{A} \mathscr{C}(n)$ and $\min \left(\left|A^{\prime}\right|,\left|B^{\prime}\right|\right)=\left|A^{\prime}\right|=\alpha^{*}$ $\mu_{k}\left(n, \alpha^{*}, \alpha^{*}\right)>\alpha^{*}-\mu_{k}\left(n, \alpha^{*}, \beta^{*}\right)=G$, contradicting the maximality of $G$.

We also claim that

$$
\mu_{k}\left(n, \alpha^{*}, \beta^{*}\right)-\alpha^{*}=\mu_{k}\left(n, \beta^{*}, \beta^{*}\right)-\beta^{*} .
$$

Otherwise, since $\mu_{k}\left(n, \alpha^{*}, \beta^{*}\right)-\alpha^{*}$ is a decreasing function of $\alpha^{*}$, we'd have $\mu_{k}\left(n, \alpha^{*}, \beta^{*}\right)-\alpha^{*}>\mu_{k}\left(n, \beta^{*}, \beta^{*}\right)-\beta^{*}$. Then with $A^{\prime}=\overleftarrow{B^{*}} \backslash \overleftarrow{B^{*}} \wedge \overrightarrow{B^{*}}$ and $B^{\prime}=$ $\overrightarrow{B^{*}} \backslash \overleftarrow{B^{*}} \wedge \overrightarrow{B^{*}}$, where $\overleftarrow{B^{*}}$ and $\overrightarrow{B^{*}}$ are respectively the first written backward and the first written forward $\beta^{*}$ elements of $[k]^{n}$, we'd have

$$
\min \left(\left|A^{\prime}\right|,\left|B^{\prime}\right|\right)=\left|A^{\prime}\right|=\beta^{*}-\mu_{k}\left(n, \beta^{*}, \beta^{*}\right)>\alpha^{*}-\mu_{k}\left(n, \alpha^{*}, \beta^{*}\right)=G
$$

again contradicting the maximality of $G$.

From (11.4) and (11.5) it follows that for every $i, 0 \leq i<\beta^{*}-\alpha^{*}$,

$$
\begin{aligned}
\mu_{k}\left(n, \alpha^{*}+i, \alpha^{*}+i+1\right) & =\mu_{k}\left(n, \alpha^{*}+i, \alpha^{*}+i\right) \quad \text { and } \\
\mu_{k}\left(n, \alpha^{*}+i+1, \alpha^{*}+i+1\right) & =\mu_{k}\left(n, \alpha^{*}+i, \alpha^{*}+i\right)+1 .
\end{aligned}
$$


Therefore, for every $i, 0 \leq i<\beta^{*}-\alpha^{*}$, the $\left(\alpha^{*}+i+1\right)$-th forward lexicographic element is simultaneously the $\left(\alpha^{*}+i+1\right)$-th backward lexicographic element, i.e.

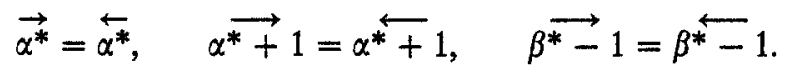

It is easy to verify, that no two consecutive integers $\alpha^{*}, \alpha^{*}+1 ; \alpha^{*}<k^{n}$; satisfy $\overrightarrow{\alpha^{*}}=\overleftarrow{\alpha^{*}}$ and $\overrightarrow{\alpha^{*}+1}=\alpha^{*}+1$. Also, we note that $\alpha^{*}+1 \leq \beta^{*}-1$, since $\beta^{*}-\alpha^{*} \geq k \geq 2$. This is a contradiction and hence $\alpha^{*}=\beta^{*}$.

\section{Theorem 6.}

(i) For $n \geq 2,2 \mid k$

$$
G(n)=\frac{k^{n}}{4} \quad \text { and } \quad f_{n}\left(\frac{k^{n}}{4}\right)=\frac{k^{n}}{4}
$$

(ii) For $n=2 m, m \geq 1,2 \nmid k$

$$
G(n)=\frac{k^{n}-1}{4} \quad \text { and } \quad f_{n}\left(\frac{k^{n}-1}{4}\right)=\frac{k^{n}-1}{4}
$$

(iii) For $n=2 m+1, m \geq 1,2 \nmid k$

$$
G(n)=\frac{k\left(k^{2 m}-1\right)}{4} \quad \text { and } \quad f_{2 m+1}\left(\frac{k\left(k^{2 m}-1\right)}{4}\right)=\frac{k\left(k^{2 m}-1\right)}{4} .
$$

Proof. (i) Recall (1.6), which was obtained by the Ahlswede/Zhang method:

$$
G(n) \leq \frac{k^{n}}{4}, \quad n \geq 2
$$

This bound is achievable for all even $k$ and all $n \geq 2$ by the following construction:

$$
\begin{aligned}
& A=\left\{\left(a_{1}, a_{2}, \ldots, a_{n}\right): a_{1} \in\left\{0,1, \ldots, \frac{k}{2}-1\right\}, a_{2} \in\left\{\frac{k}{2}, \ldots, k-1\right\}\right\}, \\
& B=\left\{\left(b_{1}, b_{2}, \ldots, b_{n}\right): b_{1} \in\left\{\frac{k}{2}, \ldots, k-1\right\}, b_{2} \in\left\{0,1, \ldots, \frac{k}{2}-1\right\}\right\} .
\end{aligned}
$$

(ii) It follows from (11.6) that $G \leq \frac{k^{2 m}-1}{4}$ and this bound is achievable by this construction:

$$
\begin{aligned}
A=\{ & \left(a_{1}, \ldots, a_{m}, a_{m+1}, \ldots, a_{2 m}\right):\left(a_{1}, \ldots, a_{m}\right) \in \underline{R}\left(\frac{k^{m}-1}{2}\right), \\
& \left.\left(a_{m+1}, \ldots, a_{2 m}\right) \in \bar{R}\left(\frac{k^{m}+1}{2}\right)\right\},
\end{aligned}
$$




$$
\begin{aligned}
B=\{ & \left(b_{1}, \ldots, b_{m}, b_{m+1}, \ldots, b_{2 m}\right):\left(b_{1}, \ldots, b_{m}\right) \in \vec{R}\left(\frac{k^{m}+1}{2}\right), \\
& \left.\left(b_{m+1}, \ldots, b_{2 m}\right) \in \underline{R}\left(\frac{k^{m}-1}{2}\right)\right\},
\end{aligned}
$$

where $\underline{R}(s)$ (resp. $\bar{R}(s)$ ) is the set of $s$ lexicographic smallest (resp. largest) elements of $[k]^{m}$.

(iii) From Lemma 9 it follows that $G(n)=\max _{\alpha}\left(\alpha-\mu_{k}(n, \alpha, \alpha)\right)$ and from Theorem 5 it follows that if $G(n)=\alpha-\mu_{k}(n, \alpha, \beta)$, then $k \mid \alpha$.

Let us denote $g(\alpha)=\alpha-\mu_{k}(n, \alpha, \alpha)$.

Lemma 10. If $\alpha<\frac{k^{m}-1}{2} k^{m+1}$ or $\alpha>\frac{k^{m}+1}{2} k^{m+1}$, then

$$
g(\alpha)<g\left(\frac{k^{m}-1}{2} k^{m+1}\right)=g\left(\frac{k^{m}+1}{2} k^{m+1}\right)=\frac{k\left(k^{2 m}-1\right)}{4} .
$$

Proof. We use from Theorem 5 in $[5] \mu_{k}(n, \alpha, \beta) \geq \frac{\alpha \cdot \beta}{k^{n}}$, from where we get

$$
g(\alpha)=\alpha-\mu_{k}(2 m+1, \alpha, \alpha) \leq \alpha-\frac{\alpha^{2}}{k^{2 m+1}}=P(\alpha), \text { say. }
$$

Also from our Theorem 2 in [10] it follows that $g(\alpha)=P(\alpha)$ if and only if $k^{m+1} \mid \alpha$.

Hence,

$$
\begin{aligned}
g\left(\frac{k^{m}-1}{2} k^{m+1}\right) & =P\left(\frac{k^{m}-1}{2} k^{m+1}\right)=\frac{k\left(k^{2 m}-1\right)}{4}=g\left(\frac{k^{m}+1}{2} k^{m+1}\right) \\
& =P\left(\frac{k^{m}+1}{2} k^{m+1}\right) .
\end{aligned}
$$

Let us note that the function $P(\alpha)$ is monotonically increasing in the interval $\left[0, \frac{k^{2 m+1}}{2}\right]$ and monotonically decreasing in $\left[\frac{k^{2 m+1}}{2}, k^{2 m+1}\right]$. Therefore, if $\alpha<$ $\frac{k^{m}-1}{2} k^{m+1}$ (or $\alpha>\frac{k^{m}+1}{2} k^{m+1}$ ), then

$$
g(\alpha) \leq P(\alpha)<P\left(\frac{k^{m}-1}{2} k^{m+1}\right)=g\left(\frac{k^{m}-1}{2} k^{m+1}\right) .
$$

So Lemma 10 shows, that $\max _{\alpha} g(\alpha)=G$ is achieved, when

$$
\frac{k^{m}-1}{2} k^{m+1} \leq \alpha \leq \frac{k^{m}+1}{2} k^{m+1} .
$$

Proof of (iii). Let us proceed by induction on $m$.

$m=1$ : We need to consider only $\alpha$ divisible by $k$ (since $(A, B)$ is bisaturated) and satisfying $\frac{k-1}{2} k^{2} \leq \alpha \leq \frac{k+1}{2} k^{2}$. 
If $\alpha=\frac{k-1}{2} k^{2}$ or $\alpha=\frac{k+1}{2} k^{2}$, then we have

$$
g\left(\frac{k-1}{2} k^{2}\right)=g\left(\frac{k+1}{2} k^{2}\right)=\frac{k\left(k^{2}-1\right)}{4} .
$$

If $\frac{k-1}{2} k^{2}<\alpha<\frac{k+1}{2} k^{2}$, then we apply Theorem 5 of [5] and get

$$
\begin{aligned}
\alpha-\mu_{k}(3, \alpha, \alpha)= & \alpha-\left[\mu_{k}\left(2, \alpha-\frac{k-1}{2} k^{2}, \frac{\alpha}{k}\right)+\frac{k-1}{2} \cdot \frac{\alpha}{k}\right] \\
= & \alpha-\left[\mu_{k}\left(1, \frac{\alpha}{k}-\frac{k-1}{2} k, \frac{\alpha}{k}-\frac{k-1}{2} k\right)+\frac{k-1}{2} \cdot \frac{\alpha}{k}\right. \\
& \left.+\frac{k-1}{2}\left(\frac{\alpha}{k}-\frac{k-1}{2} k\right)\right] \\
= & \frac{k\left(k^{2}-1\right)}{4}
\end{aligned}
$$

and $G(3)=g(\alpha)=\frac{k\left(k^{2}-1\right)}{4}$ for all $\alpha=\frac{k-1}{2} k^{2}+k \cdot s, s=0,1, \ldots, k$.

Suppose it is true for $m-1$ and for $\alpha=\frac{k^{m-1}-1}{2} k^{m}+k^{m-1} \cdot s, s=0,1, \ldots, k$; $\max _{\alpha}(\alpha-\mu(2 m-1, \alpha, \alpha))=\frac{k\left(k^{2 m-2}-1\right)}{4}$ and let us prove it for $m$.

If $\max \left(\alpha^{\prime}-\mu\left(2 m+1, \alpha^{\prime}, \alpha^{\prime}\right)\right)=\alpha-\mu(2 m+1, \alpha, \alpha)$, it follows from Lemma 10 that $\frac{k^{m}-1}{2} k^{m+1} \leq \alpha \leq \frac{k^{m}+1}{2} k^{m+1}$ and therefore $\frac{k-1}{2} k^{2 m}<\alpha<\frac{k+1}{2} k^{2 m}$.

Now

$$
\begin{aligned}
\alpha-\mu_{k}(2 m+1, \alpha, \alpha)= & \alpha-\left[\mu_{k}\left(2 m, \alpha-\frac{k-1}{2} k^{2 m}, \frac{\alpha}{k}\right)+\frac{k-1}{2} \cdot \frac{\alpha}{k}\right] \\
= & \alpha-\left[\mu_{k}\left(2 m-1, \frac{\alpha}{k}-\frac{k-1}{2} k^{2 m-1}, \frac{\alpha}{k}-\frac{k-1}{2} k^{2 m-1}\right)\right. \\
& \left.+\frac{k-1}{2} \cdot \frac{\alpha}{k}+\frac{k-1}{2}\left(\frac{\alpha}{k}-\frac{k-1}{2} k^{2 m-1}\right)\right] \\
= & \frac{\alpha}{k}-\frac{k-1}{2} k^{2 m-1}-\mu_{k}\left(2 m-1, \frac{\alpha}{k}-\frac{k-1}{2} k^{2 m-1},\right. \\
& \left.\frac{\alpha}{k}-\frac{k-1}{2} k^{2 m-1}\right)+\frac{k-1}{2} k^{2 m-1}+\frac{(k-1)^{2}}{4} k^{2 m-1} \\
= & \alpha_{1}-\mu_{k}\left(2 m-1, \alpha_{1}, \alpha_{1}\right)+\frac{k-1}{2} k^{2 m-1}+\frac{(k-1)^{2}}{4} k^{2 m-1},
\end{aligned}
$$


where $\alpha_{1}=\frac{\alpha}{k}-\frac{k-1}{2} k^{2 m-1}$, and, since $\frac{k^{m}-1}{2} k^{m+1} \leq \alpha \leq \frac{k^{m}+1}{2} k^{m+1}$ we get $\frac{k^{m-1}}{2} k^{m} \leq \alpha_{1} \leq \frac{k^{m-1}+1}{2} k^{m}$. It follows that $\alpha-\mu_{k}(2 m+1, \alpha, \alpha)$ is maximum iff $\alpha_{1}-\mu_{k}\left(2 m-1, \alpha_{1}, \alpha_{1}\right)$ is maximum. According to the induction hypothesis $\max _{\alpha_{1}}\left(\alpha_{1}-\mu_{k}\left(2 m-1, \alpha_{1}, \alpha_{1}\right)\right)=\frac{k\left(k^{2 m-2}-1\right)}{4}$ and it is achieved for $\alpha_{1}=$ $\frac{k^{m-1}-1}{2} k^{m}+k^{m-1} \cdot s ; s=0,1, \ldots, k$. Hence, $\max _{x}\left(\alpha-\mu_{k}(2 m+1, \alpha, \alpha)\right)=$ $\frac{k\left(k^{2 m}-1\right)}{4}$ and it is achieved for $\alpha=\left(\alpha_{1}+\frac{k-1}{2} k^{2 m-1}\right) k=\left(\frac{k^{m-1}-1}{2} k^{m}+k^{m-1}\right.$. $\left.s+\frac{k-1}{2} k^{2 m-1}\right) k=\frac{k^{m}-1}{2} k^{m+1}+k^{m} \cdot s ; s=0,1, \ldots, k$. Finally, we give a construction of $(A, B)$ achieving this bound:

$$
\begin{aligned}
A=\{ & \left(a_{1}, \ldots, a_{m}, a_{m+1}, \ldots, a_{2 m}, a_{2 m+1}\right):\left(a_{1}, \ldots, a_{m}\right) \in \underline{R}\left(\frac{k^{m}-1}{2}\right), \\
& \left.\left(a_{m+1}, \ldots, a_{2 m}\right) \in \bar{R}\left(\frac{k^{m}+1}{2}\right), a_{2 m+1} \in[k]\right\} \\
B= & \left\{\left(b_{1}, \ldots, b_{m}, b_{m+1}, \ldots, b_{2 m}, b_{2 m+1}\right):\left(b_{1}, \ldots, b_{m}\right) \in \bar{R}\left(\frac{k^{m}+1}{2}\right),\right. \\
& \left.\left(b_{m+1}, \ldots, b_{2 m}\right) \in \underline{R}\left(\frac{k^{m}-1}{2}\right), b_{2 m+1} \in[k]\right\} .
\end{aligned}
$$

\section{Solution of Problem IV}

Theorem 7. The function $Q(n)=\max _{(A, B) \in \mathscr{Q} \& \mathcal{Q}_{k}(n)}|A||B|$ satisfies

(i) For $n \geq 2,2 \mid k Q(n)=\frac{k^{2 n}}{16}$

(ii) For $n=2 m, m \geq 1,2 \nmid k Q(n)=\frac{\left(k^{n}-1\right)^{2}}{16}$ and this value is assumed only for $\left(A_{1}, B_{1}\right)$ and $\left(A_{2}, B_{2}\right) ;\left|A_{1}\right| \leq\left|B_{1}\right|,\left|A_{2}\right| \leq\left|B_{2}\right|$ with the cardinalities

$$
\left|A_{1}\right|=\frac{\left(k^{m}-1\right)^{2}}{4}, \quad\left|B_{1}\right|=\frac{\left(k^{m}+1\right)^{2}}{4}, \quad\left|A_{2}\right|=\frac{k^{2 m}-1}{4}, \quad\left|B_{2}\right|=\frac{k^{2 m}-1}{4} .
$$

(iii) For $n=2 m+1, m \geq 1,2 \nmid k Q(n)=\frac{\left(k^{2 m}-1\right)\left(k^{2 m+2}-1\right)}{16}$ and this value is assumed only for $\left(A_{3}, B_{3}\right)$ and $\left(A_{4}, B_{4}\right)\left|A_{3}\right| \leq\left|B_{3}\right|,\left|A_{4}\right| \leq\left|B_{4}\right|$ with the cardinalities 


$$
\begin{array}{ll}
\left|A_{3}\right|=\frac{\left(k^{m}-1\right)\left(k^{m+1}+1\right)}{4}, & \left|B_{3}\right|=\frac{\left(k^{m}+1\right)\left(k^{m+1}-1\right)}{4} \\
\left|A_{4}\right|=\frac{\left(k^{m}-1\right)\left(k^{m+1}-1\right)}{4}, & \left|B_{4}\right|=\frac{\left(k^{m}+1\right)\left(k^{m+1}+1\right)}{4} .
\end{array}
$$

Proof of (i). By the Ahlswede/Zhang method, $|A||B| \leq \frac{k^{2 n}}{16}$, so (i) follows from Theorem 6 (i). The remaining cases are more complicated. We need further auxiliary results.

Lemma 11. Let $(A, B) \in \mathscr{C} \mathscr{A} \mathscr{C}_{k}(n) ;|A| \leq|B|, n=2 m, 2 \nmid k$ and let $l$ be a positive integer.

(a) If $|A|<l^{2}<\frac{k^{2 m}-1}{4}$, then

$$
|A| \cdot \mid f_{2 m}(|A|)<l^{2} \cdot f_{2 m}\left(l^{2}\right)=l^{2} \cdot\left(k^{m}-l\right)^{2} .
$$

(b) If $l^{2}<|A| \leq \frac{k^{2 m}-1}{4}$, then

$$
|A|+\left|f_{2 m}\right|(A) \mid<l^{2}+f_{2 m}\left(l^{2}\right)=l^{2}+\left(k^{m}-l\right)^{2} .
$$

Proof. (a) We use inequality (1.8), that is,

$$
\sqrt{|A|}+\sqrt{f_{2 m}(|A|)} \leq \sqrt{k^{2 m}}
$$

Hence

$$
\sqrt{|A|}+\sqrt{f_{2 m}(|A|)} \leq \sqrt{k^{2 m}}=\sqrt{l^{2}}+\sqrt{\left(k^{m}-l\right)^{2}} .
$$

From Theorem 3 we have

$$
f_{2 m}\left(l^{2}\right)=\left(k^{m}-l\right)^{2}
$$

Therefore, from the condition

$$
|A|<l^{2}<f_{2 m}\left(l^{2}\right) \leq f_{2 m}(|A|)
$$

and (12.1) one has (a).

(b) In this case we have

$$
l^{2}<|A| \leq f_{2 m}(|A|) \leq f_{2 m}\left(l^{2}\right) .
$$

Inequality (12.1) is equivalent to

$$
|A|+\mid f_{2 m}(|A|)+2 \sqrt{|A| \cdot f_{2 m}(|A|)} \leq l^{2}+f_{2 m}\left(l^{2}\right)+2 \sqrt{l^{2} f_{2 m}\left(l^{2}\right)} .
$$

Now, if $|A|+f_{2 m}(|A|) \geq l^{2}+f_{2 m}\left(l^{2}\right)$, then from (12.2) and by arithmetic-geometric mean inequalities we have $|A| \cdot f_{2 m}(|A|)>l^{2} \cdot f_{2 m}\left(l^{2}\right)$. Hence $|A|+|B|+$ $\sqrt{|A| \cdot f_{2 m}(|A|)}>l^{2}+f_{2 m}\left(l^{2}\right)+\sqrt{l^{2} f_{2 m}\left(l^{2}\right)}$ which contradicts (12.3). 
Proof of (ii). Suppose $Q(n)$ is achieved for some pair $(A, B)$ with $|B|=f_{2 m}(|A|)$; $|A| \leq f_{2 m}(|A|)$. From (1.8) we have $|A| \leq \frac{k^{2 m}-1}{4}$ and we have also

$$
\left(\frac{k^{m}-1}{2}\right)^{2} \leq|A| \leq \frac{k^{2 m}-1}{4}
$$

because $l^{2}=\left(\frac{k^{m}-1}{2}\right)^{2}>|A|$ would contradict (a) in Lemma 11 .

If $|A|=\left(\frac{k^{m}-1}{2}\right)^{2}$, then by Theorem $3 f_{2 m}(|A|)=\left(\frac{k^{m}+1}{2}\right)^{2}$ and this gives the cardinalities $\left|A_{1}\right|,\left|B_{1}\right|$ of the Theorem.

Now, if $|A|>\left(\frac{k^{m}-1}{2}\right)^{2}$, then from Lemma 11 (b) we have

$|A|+f_{2 m}\left(\{A \mid)<\left(\frac{k^{m}-1}{2}\right)^{2}+f_{2 m}\left(\frac{k^{m}-1}{2}\right)^{2}=\left(\frac{k^{m}-1}{2}\right)^{2}+\left(\frac{k^{m}+1}{2}\right)^{2}=\frac{k^{2 m}+1}{2}\right.$

or equivalently $|A|+f_{2 m}(|A|) \leq \frac{k^{2 m}+1}{2}-1=\frac{k^{2 m}-1}{2}$, from where $|A|$. $\left|f_{2 m}(|A|)\right| \leq\left(\frac{k^{2 m}-1}{4}\right)^{2}$ with possible equality only for $|A|=f_{2 m}(|A|)=\frac{k^{2 m}-1}{4}$. However, $f_{2 m}\left(\frac{k^{2 m}-1}{4}\right)=\frac{k^{2 m}-1}{4}$ follows from Theorem 6 (ii) and this gives the cardinalities of $\left(A_{2}, B_{2}\right)$.

Clearly an $(A, B) \in \mathscr{C} \mathscr{A} \mathscr{C}_{k}(n)$ with $|A||B|=Q(n)$ is optimal and bisaturated. Therefore for some $\alpha, \beta$

$$
\alpha-\mu_{k}(n, \alpha, \beta)=|A|, \quad \beta-\mu_{k}(n, \alpha, \beta)=|B|
$$

and by Theorem 5

$$
k|\alpha, \quad k| \beta .
$$

Lemma 12. Suppose that $n=2 m+1,|A| \cdot|B|=Q(2 m+1),|A| \leq|B|$ and $|A|=$ $\alpha-\mu_{k}(2 m+1, \alpha, \beta),|B|=\beta-\mu_{k}(2 m+1, \alpha, \beta)$. Then

$$
\frac{k-1}{2} k^{2 m} \leq \alpha \leq \beta \leq \frac{k+1}{2} k^{2 m} \text {. }
$$

Proof. Let us introduce

$$
G_{2 m+1}(\alpha, \beta)=\left(\alpha-\mu_{k}(2 m+1, \alpha, \beta)\right) \cdot\left(\beta-\mu_{k}(2 m+1, \alpha, \beta)\right) .
$$

Since $\mu_{k}(2 m+1, \alpha, \beta) \geq \frac{\alpha \beta}{k^{2 m+1}}$ (by (v) Theorem 5 [5]), stated in Section 2, we have

$$
\begin{aligned}
G_{2 m+1}(\alpha, \beta) & \left.\leq\left(\alpha-\frac{\alpha \beta}{k^{2 m+1}}\right) \beta-\frac{\alpha \beta}{k^{2 m+1}}\right)=\alpha\left(1-\frac{\alpha}{k^{2 m+1}}\right) \cdot \beta\left(1-\frac{\beta}{k^{2 m+1}}\right) \\
& =P(\alpha, \beta), \text { say. }
\end{aligned}
$$


It is easy to see that

$$
P\left(\alpha_{1}, \beta\right)<P\left(\alpha_{2}, \beta\right), \quad \text { if } \quad \alpha_{1}<\alpha_{2}<\frac{k^{2 m+1}}{2} \text { or } \alpha_{1}>\alpha_{2}>\frac{k^{2 m+1}}{2}
$$

and

$$
P\left(\alpha, \beta_{1}\right)<P\left(\alpha, \beta_{2}\right), \quad \text { if } \quad \beta_{1}<\beta_{2}<\frac{k^{2 m+1}}{2} \text { or } \beta_{1}>\beta_{2}>\frac{k^{2 m+1}}{2} \text {. }
$$

Suppose that $\alpha<\frac{k-1}{2} k^{2 m}$ (or $\left.\alpha>\frac{k+1}{2} k^{2 m}\right)$, then

$$
Q(2 m+1)=G_{2 m+1}(\alpha, \beta) \leq P(\alpha, \beta)<P\left(\frac{k-1}{2} k^{2 m}, \beta\right) .
$$

On the other hand since $k \mid \beta$ by (12.5), then $P\left(\frac{k-1}{2} k^{2 m}, \beta\right)=$ $G_{2 m+1}\left(\frac{k-1}{2} k^{2 m}, \beta\right)$, (we apply Theorem 2 of $[10]$ ) and hence $Q(2 m+1)<$ $G_{2 m+1}\left(\frac{k-1}{2} k^{2 m}, \beta\right)$, which contradicts maximality.

Proof of (iii). Let us proceed by induction on $m$.

$\underline{m=1:}$ From Lemma 12 one has

$$
\frac{k-1}{2} k^{2} \leq \alpha \leq \beta \leq \frac{k+1}{2} k^{2} .
$$

Case. $\alpha=\frac{k-1}{2} k^{2}$ (or $\left.\beta=\frac{k+1}{2} k^{2}\right)$.

Since $k^{2} \mid \alpha$ and $k \mid \beta$, we have $\mu_{k}\left(3, \frac{k-1}{2} k^{2}, \beta\right)=\frac{k-1}{2} \cdot \frac{\beta}{k}$ and

$$
G_{3}\left(\frac{k-1}{2} k^{2}, \beta\right)=\left(\frac{k-1}{2} k^{2}-\frac{k-1}{2} \frac{\beta}{k}\right)\left(\beta-\frac{k-1}{2} \frac{\beta}{k}\right)=\frac{\left(k^{2}-1\right)}{4 k} \beta\left(k^{2}-\frac{\beta}{k}\right) \text {. }
$$

Let us consider the function $T(\beta)=\beta\left(k^{2}-\frac{\beta}{k}\right)$. We verify that $T\left(\beta_{1}\right)<T\left(\beta_{2}\right)$ if $\beta_{1}<\beta_{2}<\frac{k^{3}}{2}$ or $\beta_{1}>\beta_{2}>\frac{k^{3}}{2}$.

Hence, taking account of $2 \nmid k, k \mid \beta$ one has $\max _{k \mid \beta, 2\} k} T(\beta)=\frac{k\left(k^{2}-1\right)\left(k^{2}+1\right)}{4}$ and the maximum is achieved for $\beta_{1}=\frac{k^{3}-k}{2}, \beta_{2}=\frac{k^{3}+k}{2}$ only. 


$$
\begin{aligned}
& \text { So, } \max _{\beta} G_{3}\left(\frac{k-1}{2} k^{2}, \beta\right)=\frac{\left(k^{2}-1\right)\left(k^{4}-1\right)}{16} \text { and } \\
& \qquad \begin{array}{l}
\left|A_{3}\right|=\frac{k-1}{2} k^{2}-\mu_{k}\left(3, \frac{k-1}{2} k^{2}, \beta_{1}\right)=\frac{(k-1)\left(k^{2}+1\right)}{4} \\
\left|B_{3}\right|=\beta_{1}-\mu_{k}\left(3, \frac{k-1}{2} k^{2}, \beta_{1}\right)=\frac{(k+1)\left(k^{2}-1\right)}{4}
\end{array}
\end{aligned}
$$

or

$$
\left\{\begin{array}{l}
\left|A_{4}\right|=\frac{k-1}{2} k^{2}-\mu_{k}\left(3, \frac{k-1}{2} k^{2}, \beta_{2}\right)=\frac{(k-1)\left(k^{2}-1\right)}{4} \\
\left|B_{4}\right|=\beta_{2}-\mu_{k}\left(3, \frac{k-1}{2} k^{2}, \beta_{2}\right)=\frac{(k+1)\left(k^{2}+1\right)}{4}
\end{array}\right.
$$

Case. $\frac{k-1}{2} k^{2}<\alpha \leq \beta<\frac{k+1}{2} k^{2}$

We apply Theorem 5 of [5]:

$$
\begin{aligned}
\mu_{k}(3, \alpha, \beta)= & \mu_{k}\left(2, \alpha-\frac{k-1}{2} k^{2}, \frac{\beta}{k}\right)+\frac{(k-1)}{2} \frac{\beta}{k} \\
= & \mu_{k}\left(1, \frac{\alpha}{k}-\frac{k-1}{2} k, \frac{\beta}{k}-\frac{k-1}{2} k\right) \\
& +\frac{(k-1)}{2} \frac{\beta}{k}+\frac{(k-1)}{2} \frac{\alpha}{k}-\frac{(k-1)(k-1) k}{4} \\
= & \frac{\alpha}{k}-\frac{k-1}{2} k+\frac{k-1}{2} \frac{\beta}{k}+\frac{k-1}{2} \frac{\alpha}{k}-\frac{(k-1)^{2} k}{4} \\
= & \alpha \cdot \frac{k+1}{2 k}+\beta \frac{(k-1)}{2 k}-\frac{k\left(k^{2}-1\right)}{4}
\end{aligned}
$$

and hence

$$
\begin{aligned}
G_{3}(\alpha, \beta) & =\left(\alpha-\mu_{k}(3, \alpha, \beta)\right)\left(\beta-\mu_{k}(3, \alpha, \beta)\right) \\
& =\left(\frac{k-1}{2 k} \alpha-\frac{k-1}{2 k} \beta+\frac{k\left(k^{2}-1\right)}{4}\right) \cdot\left(\frac{k+1}{2 k} \beta-\frac{k+1}{2 k} \alpha+\frac{k\left(k^{2}-1\right)}{4}\right) \\
& =\frac{k^{2}\left(k^{2}-1\right)^{2}}{16}+\frac{\left(k^{2}-1\right)}{4}(\beta-\alpha)\left(1-\frac{\beta-\alpha}{k^{2}}\right) .
\end{aligned}
$$

Now let us define $\beta-\alpha=x$ and consider the function $L(x)=x\left(1-\frac{x}{k^{2}}\right)$. We verify that $L\left(x_{1}\right)<L\left(x_{2}\right)$, if $x_{1}<x_{2}<\frac{k^{2}}{2}$ or $x_{1}>x_{2}>\frac{k^{2}}{2}$. With regards to $2 \nmid k$, 
$k|\alpha, k| \beta$ one has $\max _{k \mid x} L(x)=\frac{k^{2}-k}{2}\left(1-\frac{k^{2}-k}{2 k^{2}}\right)=\frac{(k-1)(k+1)}{4}$ and it is achieved only for $x_{1}=\frac{k^{2}-k}{2}, x_{2}=\frac{k^{2}+k}{2}$. Therefore from (12.9)

$$
G_{3}(\alpha, \beta)=\frac{k^{2}\left(k^{2}-1\right)^{2}}{16}+\frac{\left(k^{2}-1\right)(k-1)(k+1)}{4 \cdot 4}=\frac{\left(k^{2}-1\right)\left(k^{4}-1\right)}{16} .
$$

Furthermore, substitution of $\beta-\alpha=x_{1}$ (or $\beta-\alpha=x_{2}$ ) in (12.8) give us

$$
\mu_{k}(3, \alpha, \beta)=\alpha-\frac{(k-1)\left(k^{2}+1\right)}{4} \quad \text { or } \quad \alpha-\frac{(k-1)\left(k^{2}-1\right)}{4}
$$

and hence the same parameters for $\left(A_{3}, B_{3}\right)$ and $\left(A_{4}, B_{4}\right)$ occur. Now suppose that the statement is true for $n=2 m-1$ that is $Q_{2 m-1}=\frac{\left(k^{2 m-2}-1\right)\left(k^{2 m}-1\right)}{16}$ and it assumed only for

$$
\left(A_{3}^{\prime}, B_{3}^{\prime}\right),\left(A_{4}^{\prime}, B_{4}^{\prime}\right):\left\{\begin{array} { l } 
{ | A _ { 3 } ^ { \prime } | = \frac { ( k ^ { m - 1 } - 1 ) ( k ^ { m } + 1 ) } { 4 } } \\
{ | B _ { 3 } ^ { \prime } | = \frac { ( k ^ { m - 1 } + 1 ) ( k ^ { m } - 1 ) } { 4 } }
\end{array} \quad \left\{\begin{array}{l}
\left|A_{4}^{\prime}\right|=\frac{\left(k^{m-1}-1\right)\left(k^{m}-1\right)}{4} \\
\left|B_{4}^{\prime}\right|=\frac{\left(k^{m-1}+1\right)\left(k^{m}+1\right)}{4}
\end{array} .\right.\right.
$$

According to Lemma 12 one has

$$
\frac{k-1}{2} k^{2 m} \leq \alpha \leq \beta \leq \frac{k+1}{2} k^{2 m}
$$

Case. $\alpha=\frac{k-1}{2} k^{2 m}\left(\right.$ or $\left.\beta=\frac{k+1}{2} k^{2 m}\right)$

Since $k^{2 m}|\alpha, k| \beta$ then $\mu_{k}\left(\frac{k-1}{2} k^{2 m}, \beta\right)=\frac{(k-1) \beta}{k}$ and $G_{2 m+1}\left(\frac{k-1}{2} k^{2 m}, \beta\right)=$ $\left(\frac{(k-1)}{2} k^{2 m}-\frac{(k-1)}{2} \frac{\beta}{k}\right)\left(\beta-\frac{(k-1)}{2} \frac{\beta}{k}\right)=\frac{(k-1)(k+1)}{4 k} \cdot \beta\left(k^{2 m}-\frac{\beta}{k}\right)$. We verify that $\max G_{2 m+1}\left(\frac{k-1}{2} k^{2 m}, \beta\right)$ is assumed for $\beta_{1}=\frac{k^{2 m+1}-k}{2}$ or $\beta_{2}=\frac{k^{2 m+1}+k}{2}$ and $G_{2 m+1}\left(\frac{k-1}{2} k^{2 m}, \beta_{1}\right)=G_{2 m+1}\left(\frac{k-1}{2} k^{2 m}, \beta_{2}\right)=\frac{\left(k^{2 m}-1\right)\left(k^{2 m}+1\right)\left(k^{2}-1\right)}{16}<$ $\frac{\left(k^{2 m}-1\right)\left(k^{2 m+2}-1\right)}{16}$, if $m>1$ and so $\alpha>\frac{k-1}{2} k^{2 m}$.

Case. $\frac{k-1}{2} k^{2 m}<\alpha \leq \beta<\frac{k+1}{2} k^{2 m}$

We apply again Theorem 5 of [5]: 


$$
\begin{aligned}
\mu_{k}(2 m+1, \alpha, \beta)= & \mu_{k}\left(2 m, \alpha-\frac{k-1}{2} k^{2 m}, \frac{\beta}{k}\right)+\frac{k-1}{2} \frac{\beta}{k} \\
= & \mu_{k}\left(2 m-1, \frac{\alpha}{k}-\frac{k-1}{2} k^{2 m-1}, \frac{\beta}{k}-\frac{k-1}{2} k^{2 m-1}\right) \\
& +\frac{k-1}{2} \frac{\beta}{k}+\frac{k-1}{2} \frac{\alpha}{k}-\frac{(k-1)^{2}}{4} k^{2 m-1}
\end{aligned}
$$

and so

$$
\begin{aligned}
\alpha-\mu_{k}(2 m+1, \alpha, \beta)= & \frac{k+1}{2}\left(\alpha_{1}-\mu_{k}\left(2 m-1, \alpha_{1}, \beta_{1}\right)\right) \\
& -\frac{(k-1)}{2}\left(\beta_{1}-\mu_{k}\left(2 m-1, \alpha_{1}, \beta_{1}\right)\right)+\frac{\left(k^{2}-1\right)}{4} k^{2 m-1}
\end{aligned}
$$

and

$$
\begin{aligned}
\beta-\mu_{k}(2 m+1, \alpha, \beta)= & \frac{(k+1)}{2}\left(\beta_{1}-\mu_{k}\left(2 m-1, \alpha_{1}, \beta_{1}\right)\right) \\
& -\frac{(k-1)}{2}\left(\alpha_{1}-\mu_{k}\left(2 m-1, \alpha_{1}, \beta_{1}\right)\right)+\frac{\left(k^{2}-1\right)}{4} k^{2 m-1},
\end{aligned}
$$

where $\alpha_{1}=\frac{\alpha}{k}-\frac{(k-1)}{2} k^{2 m-1}, \beta_{1}=\frac{\beta}{k}-\frac{k-1}{2} k^{2 m-1}$.

Substituting (12.11) in $G_{2 m+1}(\alpha, \beta)$, after simplification one has

$$
\begin{aligned}
G_{2 m+1}(\alpha, \beta)= & G_{2 m-1}\left(\alpha_{1}, \beta_{1}\right) \cdot k^{2}+\frac{\left(k^{2}-1\right)^{2}}{16} k^{4 m-2} \\
& +\frac{\left(k^{2}-1\right)}{4}\left(\alpha_{1}+\beta_{1}-2 \mu_{k}\left(2 m-1, \alpha_{1}, \beta_{1}\right)\right) \\
& \cdot\left(k^{2 m-1}-\left(\alpha_{1}+\beta_{1}-2 \mu_{k}\left(2 m-1, \alpha_{1}, \beta_{1}\right)\right)\right) .
\end{aligned}
$$

Therefore,

$$
\max G_{2 m+1}(\alpha, \beta) \leq \frac{\left(k^{2}-1\right)^{2}}{16} k^{4 m-2}+\max G_{2 m-1}\left(\alpha_{1}, \beta_{1}\right) \cdot k^{2}+\frac{k^{2}-1}{4} \cdot \max _{y} R(y),
$$

where $y=\alpha_{1}+\beta_{1}-2 \mu_{k}\left(2 m-1, \alpha_{1}, \beta_{1}\right)$ and $R(y)=y\left(k^{2 m-1}-y\right)$. By the induction hypothesis $\max G_{2 m-1}\left(\alpha_{1}, \beta_{1}\right)=\frac{\left(k^{2 m-2}-1\right)\left(k^{2 m}-1\right)}{16}$ and is achieved only for (see (12.10))

$$
\left\{\begin{array} { l } 
{ \alpha _ { 1 } ^ { \prime } - \mu _ { k } ( 2 m - 1 , \alpha _ { 1 } ^ { \prime } , \beta _ { 1 } ^ { \prime } ) = | A _ { 3 } ^ { \prime } | } \\
{ \beta _ { 1 } ^ { \prime } - \mu _ { k } ( 2 m - 1 , \alpha _ { 1 } ^ { \prime } , \beta _ { 1 } ^ { \prime } ) = | B _ { 3 } ^ { \prime } | }
\end{array} \text { and } \left\{\begin{array}{l}
\alpha_{1}^{\prime \prime}-\mu_{k}\left(2 m-1, \alpha_{1}^{\prime \prime}, \beta_{1}^{\prime \prime}\right)=\left|A_{4}^{\prime}\right| \\
\beta_{1}^{\prime \prime}-\mu_{k}\left(2 m-1, \alpha_{1}^{\prime \prime}, \beta_{1}^{\prime \prime}\right)=\left|B_{4}^{\prime}\right|
\end{array}\right.\right. \text {. }
$$


Also we verify that $\max _{y} R(y)=\frac{\left(k^{2 m-1}-1\right)\left(k^{2 m-1}+1\right)}{4}$ and the maximum is assumed for $y_{1}=\frac{k^{2 m-1}-1}{2}, y_{2}=\frac{k^{2 m-1}+1}{2}$ and they are compatible with (12.12).

Hence

$$
\begin{aligned}
G_{2 m+1}(\alpha, \beta) \leq & \frac{k^{2}\left(k^{2 m-2}-1\right)\left(k^{2 m}-1\right)}{16}+\frac{\left(k^{2}-1\right)^{2} k^{4 m-2}}{16} \\
& +\frac{\left(k^{2}-1\right)\left(k^{2 m-1}-1\right)\left(k^{2 m-1}+1\right)}{16}=\frac{\left(k^{2 m}-1\right)\left(k^{2 m+2}-1\right)}{16} .
\end{aligned}
$$

On the other hand we take $\alpha^{*}=\left(\alpha_{1}+\frac{k-1}{2} k^{2 m-1}\right) k, \beta=\left(\beta_{1}+\frac{k-1}{2} k^{2 m-1}\right) k$ and verify $G_{2 m+1}(\alpha, \beta)=\frac{\left(k^{2 m}-1\right)\left(k^{2 m+2}-1\right)}{16}$.

The optimal constructions are:

$$
\begin{aligned}
& A_{3}=\left\{\left(a_{1}, \ldots, a_{m}, a_{m+1}, \ldots, a_{2 m+1}\right):\left(a_{1}, \ldots, a_{m}\right) \in \underline{R}_{m}\left(\frac{k^{m}-1}{2}\right),\right. \\
&\left.\left(a_{m+1}, \ldots, a_{2 m+1}\right) \in \bar{R}_{m+1}\left(\frac{k^{m+1}+1}{2}\right)\right\} \\
& B_{3}=\left\{\left(b_{1}, \ldots, b_{m}, b_{m+1}, \ldots, b_{2 m+1}\right):\left(b_{1}, \ldots, b_{m}\right) \in \bar{R}_{m}\left(\frac{k^{m}+1}{2}\right),\right. \\
&\left.\left(b_{m+1}, \ldots, b_{2 m+1}\right) \in \underline{R}_{m+1}\left(\frac{k^{m+1}-1}{2}\right)\right\}
\end{aligned}
$$

and

$$
\begin{aligned}
A_{4}= & \left\{\left(a_{1}, \ldots, a_{m}, a_{m+1}, \ldots, a_{2 m+1}\right):\left(a_{1}, \ldots, a_{m}\right) \in \underline{R}_{m}\left(\frac{k^{m}-1}{2}\right),\right. \\
& \left.\left(a_{m+1}, \ldots, a_{2 m+1}\right) \in \bar{R}_{m+1}\left(\frac{k^{m+1}-1}{2}\right)\right\} \\
B_{4}= & \left\{\left(b_{1}, \ldots, b_{m}, b_{m+1}, \ldots, b_{2 m+1}\right):\left(b_{1}, \ldots, b_{m}\right) \in \bar{R}_{m}\left(\frac{k^{m}+1}{2}\right),\right. \\
& \left.\left(b_{m+1}, \ldots, b_{2 m+1}\right) \in \underline{R}_{m+1}\left(\frac{k^{m+1}+1}{2}\right)\right\} .
\end{aligned}
$$

\section{Solution of Problem V}

From Lemma 4 in Section 4 we conclude that

$$
a_{\Delta}(n)=\max _{0 \leq \alpha \leq k^{n}}\left(\alpha-\mu_{k}(n, \alpha, \alpha+\Delta)\right) \text {. }
$$


It is convenient to introduce the function

$$
F_{n}(\alpha, \beta)=\alpha-\mu_{k}(n, \alpha, \beta)
$$

and write for any $\Delta,-k^{n} \leq \Delta \leq k^{n}$

$$
a_{\Delta}(n)=\max _{\alpha} F_{n}(\alpha, \alpha+\Delta) \text {. }
$$

\section{Theorem 8.}

(i) $n=2 m$ : For any $\Delta$ and $t$ with $-k^{2 m} \leq k^{2 m}-2 t k^{m}-k^{m} \leq \Delta \leq k^{2 m}-2 t k^{m}+$ $k^{m} \leq k^{2 m}$

$$
a_{\Delta}(n)=F_{2 m}\left(t k^{m}, t k^{m}+\Delta\right) .
$$

(ii) $n=2 m+1$ : For any $\Delta$ and $t$ with $-k^{2 m+1} \leq k^{2 m+1}-2 t k^{m+1}-k^{m+1} \leq \Delta \leq$ $k^{2 m+1}-2 t k^{m+1}+k^{m+1} \leq k^{2 m+1}$

$$
a_{\Delta}(n)=F_{2 m+1}\left(t k^{m+1}, t k^{m+1}+\Delta\right) .
$$

The proof uses three auxiliary results (Lemmas 13,14 , and 15 below) concerning the function $F_{n}$, which are obtained by a thorough analysis. Essential use is made of properties of the function $\mu_{k}$, which were obtained by Daykin, Kleitman, and West. We rely upon their Theorem 5 in [5], which is restated in Section 2 and from now on will be refered to as Theorem DKW.

We present and prove now our auxiliary results.

Lemma 13. Assume that $0<s<k, \alpha \leq s \cdot k^{n-1}$, and $\beta \leq(k-s) k^{n-1}$. Then

(i) $F_{n}(\alpha, \beta) \geq F_{n}(\alpha-r k, \beta-r k)$ for all $r ; 0 \leq r \leq \frac{\min (\alpha, \beta)}{k}$.

(ii) If $k \mid \alpha$, then $F_{n}(\alpha, \beta) \geq F_{n}(\alpha-\delta, \beta-\delta)$ for all $\delta ; 0 \leq \delta \leq \min (\alpha, \beta)$.

Proof. (i) From Theorem 4 in [5] (see Section 2) it follows that $\mu_{k}(n, \alpha, \beta)$ can be assumed for a pair of ideals $A, B$ with $|A|=\alpha,|B|=\beta$, where $A$ are the $\alpha$ smallest and written backwards, $B$ are the $\beta$ smallest $n$-digit $k$-ary numbers. Further, we continue to denote by $\bar{x}$ (resp. $\vec{x}$ ) the $x$-th lexicographic vector written backwards (resp. the $x$-th lexicographic vector).

Now let

$$
\begin{aligned}
& A=A_{1} \cup A_{2} \text { with }\left|A_{1}\right|=\alpha-r k, \quad\left|A_{2}\right|=r k \quad \text { and let } \\
& B=B_{1} \cup B_{2} \quad \text { with } \quad\left|B_{1}\right|=\beta-r k \quad \text { and } \quad\left|B_{2}\right|=r k,
\end{aligned}
$$

where $A_{1}$ (resp. $B_{1}$ ) is the set of the $(\alpha-r k)$ smallest lexicographic vectors written backwards (resp. $(\beta-r k)$ smallest lexicographic vectors). Then $F_{n}(\alpha, \beta)=$ $\left|A_{1} \cup A_{2}\right|-\left|\left(A_{1} \cup A_{2}\right) \cap\left(B_{1} \cup B_{2}\right)\right|=\left|A_{1}\right|+\left|A_{2}\right|-\left|A_{1} \cap B_{1}\right|-\left|A_{1} \cap B_{2}\right|-$ $\left|A_{2} \cap\left(B_{1} \cup B_{2}\right)\right|$ and $F_{n}(\alpha-r k, \beta-r k)=\left|A_{1}\right|-\left|A_{1} \cap B_{1}\right|$.

We notice in view of the bound on $\alpha$ that the last component of a vector in the ideal $A$ is never one of the $(k-s)$ integers $s, s+1, \ldots, k-1$ while each of the digits $0,1, \ldots, k-1$ must occur exactly $\frac{\left|B_{2}\right|}{k}=r$ times in the last component of a 
vector in $B_{2}$ because the vectors in $B_{2}$ are consecutive. It follows that $\left|A_{1} \cap B_{2}\right| \leq$ $\left|B_{2}\right|-r(k-s)$. Similarly, in view of the bound on $\beta$, it follows that the first component of a vector in the ideal $B$ is never one of the $s$ digits $k-s, k-s+1$, $\ldots, k-1$ while each of the digits $0,1, \ldots, k-1$ must occur exactly $\frac{\left|A_{2}\right|}{k}=r$ times as a first component of a vector in $A_{2}$ since the vectors in $A_{2}$ are consecutive (in the backward order). It follows that $\left|A_{2} \cap\left(B_{1} \cup B_{2}\right)\right| \leq\left|A_{2}\right|-r s$ and therefore $F_{n}(\alpha, \beta) \geq\left|A_{1}\right|-\left|A_{1} \cap B_{1}\right|=F_{n}(\alpha-r k, \beta-r k)$.

(ii) Suppose that $A=A_{1}^{*} \cup A_{2}^{*}$ with $\left|A_{1}^{*}\right|=\alpha-\delta,\left|A_{2}^{*}\right|=\delta$ and $B=B_{1}^{*} \cup B_{2}^{*}$ with $\left|B_{1}^{*}\right|=\beta-\delta$ and $\left|B_{2}^{*}\right|=\delta$, where $A_{1}^{*}$ (resp. $\left.B_{1}^{*}\right)$ is the set of $(\alpha-\delta)$ smallest lexicographic vectors written backwards (resp. $(\alpha-\delta)$ smallest lexicographic vectors). From (i) it follows that it is sufficient to consider $\delta ; 0<\delta<k$.

We consider the first components of vectors $A_{2}^{*}$. As $k \mid \alpha$ the first component of the $\alpha$-th vector is equal to $k-1$. Hence, the first components of vectors $A_{2}^{*}$ are $k-\delta, k-\delta+1, \ldots, k-1$.

We consider separately cases a) $\delta \leq s$ and b) $\delta>s$.

a) $\delta \leq s$ : Then $A_{2}^{*} \cap\left(B_{1}^{*} \cup B_{2}^{*}\right)=\varnothing$, since $\beta \leq(k-s) k^{n-1}$ implies that the first components of vectors $B=B_{1}^{*} \cup B_{2}^{*}$ are smaller than or equal to $k-s-1<$ $k-s \leq k-\delta$. Also, clearly $\left|A_{1}^{*} \cap B_{2}^{*}\right| \leq\left|B_{2}^{*}\right|=\left|A_{2}^{*}\right|=\delta$. Therefore, $F_{n}(\alpha, \beta)=$ $\left|A_{1}^{*} \cup A_{2}^{*}\right|-\left|\left(A_{1}^{*} \cup A_{2}^{*}\right) \cap\left(B_{1}^{*} \cup B_{2}^{*}\right)\right|=\left|A_{1}^{*}\right|-\left|A_{1}^{*} \cap B_{1}^{*}\right|+\left|A_{2}^{*}\right|-\left|A_{1}^{*} \cap B_{2}^{*}\right|-$ $\left|A_{2}^{*} \cap\left(B_{1}^{*} \cup B_{2}^{*}\right)\right| \geq\left|A_{1}^{*}\right|-\left|A_{1}^{*} \cap B_{1}^{*}\right|=F_{n}(\alpha-\delta, \beta-\delta)$.

b) $\underline{\delta>s}$ : Then $\left|A_{2}^{*} \cap\left(B_{1}^{*} \cup B_{2}^{*}\right)\right| \leq \delta-s$ since the first components of the $\delta$ vectors in $A_{2}^{*}$ are $k-\delta, k-\delta+1, \ldots, k-1$ while the first components of vectors in $B$ are $\leq k-s-1$ in view of $\beta \leq(k-s) k^{n-1}$. Now suppose that the last components of the $\delta$ vectors in $B_{2}^{*}$ are $i, i+1, \ldots, i+\delta-1(\bmod k)$. These integers are distinct because $\delta<k$. It follows that $\left|A_{1}^{*} \cap B_{2}^{*}\right| \leq s$ since otherwise some vector in $A^{*}$ would have last coordinate $\geq s$, which would imply $\left|A_{1}^{*}\right| \geq(s+1) k^{n+1}$, contradicting that $\left|A_{1}^{*}\right|=\alpha-\delta<\alpha \leq s k^{n-1}$. Therefore, $F_{n}(\alpha, \beta)=\left|A_{1}^{*}\right|$ $\left|A_{1}^{*} \cap B_{1}^{*}\right|+\left|A_{2}^{*}\right|-\left|A_{1}^{*} \cap B_{2}^{*}\right|-\left|A_{2}^{*} \cap\left(B_{1}^{*} \cup B_{2}^{*}\right)\right| \geq\left|A_{1}^{*}\right|-\left|A_{1}^{*} \cap B_{1}^{*}\right|+\delta-$ $(\delta-s)-s=\left|A_{1}^{*}\right|-\left|A_{1}^{*} \cap B_{1}^{*}\right|=F_{n}(\alpha-\delta, \beta-\delta)$.

Corollary. Let $0<s<k ; \alpha \geq s k^{n-1}$ and $\beta \geq(k-s) k^{n-1}$, then

(i) $F_{n}(\alpha, \beta) \geq F_{n}(\alpha+r k, \beta+r k)$

(ii) $k \mid \alpha$ implies $F_{n}(\alpha, \beta) \geq F_{n}(\alpha+\delta, \beta+\delta)$.

Proof. We use the identity (iv) of Theorem DKW, that is $\mu_{k}\left(n, k^{n}-\alpha, k^{n}-\beta\right)=$ $\mu_{k}(n, \alpha, \beta)+k^{n}-\alpha-\beta$. One has $F_{n}(\alpha, \beta)=\alpha-\mu_{k}(n, \alpha, \beta)=\alpha-\mu_{k}\left(n, k^{n}-\alpha\right.$, $\left.k^{n}-\beta\right)+k^{n}-\alpha-\beta=k^{n}-\beta-\mu_{k}\left(n, k^{n}-\beta, k^{n}-\alpha\right)=F_{n}\left(k^{n}-\beta, k^{n}-\alpha\right)$ and $F_{n}(\alpha+r k, \beta+r k)=F_{n}\left(k^{n}-\beta-r k, k^{n}-\alpha-r k\right)$.

We introduce $\alpha^{*}=k^{n}-\beta$ and $\beta^{*}=k^{n}-\alpha$ and observe that $\alpha^{*} \leq s \cdot k^{n-1}$, $\beta^{*} \leq(k-s) k^{n-1}$. According to (i) in Lemma 13 one has $F_{n}(\alpha, \beta)=F_{n}\left(k^{n}-\beta\right.$, $\left.k^{n}-\alpha\right)=F_{n}\left(\alpha^{*}, \beta^{*}\right) \geq F_{n}\left(\alpha^{*}-r k, \beta^{*}-r k\right)=F_{n}\left(k^{n}-\beta-r k, k^{n}-\alpha-r k\right)=$ $F_{n}(\alpha+r k, \beta+r k)$.

Analogously one can prove (ii). 
Lemma 14. Assume that $k \gamma \alpha$ and $\alpha \equiv \alpha_{1} \bmod k, 0<\alpha_{1}<k$. Then $F_{n}(\alpha, \beta) \leq$ $\max \left\{F_{n}\left(\alpha-\alpha_{1}, \beta-\alpha_{1}\right), F_{n}\left(\alpha+k-\alpha_{1}, \beta+k-\alpha_{1}\right)\right\}$.

Proof. As in the proof of Lemma 13 let $A,|A|=\alpha$, be the ideal of the $\alpha$ smallest lexicographic vectors written backwards, and $B,|B|=\beta$, be the $\beta$ smallest $n$-digit $k$-ary vectors. The first component of $\alpha-1$, being biggest vector in $A$, is equal to $\alpha_{1}-1$, since $\alpha=\alpha_{1}(\bmod k)$. We distinguish two cases: $\alpha-1 \in B$ and $\alpha-1 \notin B$.

1) $\alpha-1 \in B$ : Then $\alpha \overleftarrow{-1}=\vec{\gamma}$ for some $\gamma<\beta$. It is easy to see, that $\alpha-i=$

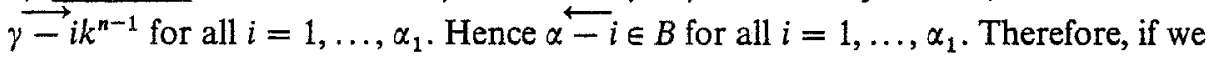
remove the last $\alpha_{1}$ vectors from $A$, we decrease $A \cap B$ by $\alpha_{1}$. This is equivalent to $\mu_{k}(n, \alpha, \beta)=\mu_{k}\left(n, \alpha-\alpha_{1}, \beta\right)+\alpha_{1} \geq \mu_{k}\left(n, \alpha-\alpha_{1}, \beta-\alpha_{1}\right)+\alpha_{1}$, from where one has $F_{n}\left(\alpha-\alpha_{1}, \beta-\beta_{1}\right)=\alpha-\alpha_{1}-\mu_{k}\left(n, \alpha-\alpha_{1}, \beta-\beta_{1}\right) \geq \alpha-\mu_{k}(n, \alpha, \beta)=F_{n}(\alpha, \beta)$.

2) $\underline{\leftarrow-1} \notin B$ : Then clearly $\alpha+i \notin B, i=0, \ldots, k-\alpha_{1}-1$. We add the next $\left(k-\alpha_{1}\right)$ lexicographic vectors to the ideals $A$ and $B: A^{*}=A \cup B^{\prime}, B^{*}=B \cup B^{\prime}$; $A^{\prime}=\{\bar{\alpha}, \ldots, \alpha+k \stackrel{-\alpha}{-\alpha}-1\}, \quad B^{\prime}=\left\{\vec{\beta}, \ldots, \beta+\overrightarrow{k-\alpha_{1}}-1\right\}$. Let us consider $A^{*} \cap B^{*}=\left(A^{*} \cap B\right) \cup\left(A^{*} \cap B^{\prime}\right)$. As $\alpha+i \notin B$ for $i=0, \ldots, k-\alpha_{1}-1$, then

$$
A^{*} \cap B=A \cap B \text {. }
$$

Also, obviously $\left|A^{*} \cap B^{\prime}\right| \leq\left|B^{\prime}\right|=k-\alpha_{1}$. Therefore, $\mu_{k}\left(n, \alpha+k-\alpha_{1}, \beta+k-\alpha_{1}\right)$ $=\left|A^{*} \cap B^{*}\right|=\left|A^{*} \cap B\right|+\left|A^{*} \cap B^{\prime}\right|=|A \cap B|+\left|A^{*} \cap B^{\prime}\right|=\mu_{k}(n, \alpha, \beta)+$ $\left|A^{*} \cap B^{*}\right| \leq \mu_{k}(n, \alpha, \beta)+k-\alpha_{1}$ or equivalently $F_{n}\left(\alpha+k-\alpha_{1}, \beta+k-\alpha_{1}\right)=$ $\alpha+k-\alpha_{1}-\mu_{k}\left(n, \alpha+k-\alpha_{1}, \beta+k-\alpha_{1}\right) \geq \alpha-\mu_{k}(n, \alpha, \beta)=F_{n}(\alpha, \beta)$.

Now we try to estimate $\max _{\alpha} F_{n}(\alpha, \alpha+\Delta)$ for arbitrary fixed $\Delta$. We concentrate on the case $n=2 m$. Obviously, for any $\Delta$, there exists a unique $t$, such that

$$
k^{2 m}-2 k^{m} t-k^{m}<\Delta \leq k^{2 m}-2 k^{m} t+k^{m} \text {. }
$$

Lemma 15. (i) If $s k^{m-1}<t<(s+1) k^{m-1}$, then $\max _{\alpha} f_{2 m}(\alpha, \alpha+\Delta)$ can be attained when

$k \mid \alpha, \quad s k^{2 m-1} \leq \alpha \leq(s+1) k^{2 m-1}$ and $(k-s-1) k^{2 m-1} \leq \alpha+\Delta \leq(k-s) k^{2 m+1}$.

(ii) If $t=s k^{m-1}$, then in case

(a) $k^{2 m}-2 k^{m} t<\Delta \leq k^{2 m}-2 k^{m} t+k^{m}$

$\max _{\alpha} f_{2 m}(\alpha, \alpha+\Delta)$ can be attained, when

$$
\begin{gathered}
k \mid \alpha, \quad s k^{2 m-1}-k^{m} \leq \alpha \leq s k^{2 m-1} \text { and } \\
(k-s) k^{2 m-1} \leq \alpha+\Delta \leq(k-s) k^{2 m-1}+k^{m} .
\end{gathered}
$$

and in case

(b) $k^{2 m}-2 k^{m} t-k^{m}<\Delta \leq k^{2 m}-k^{m} t$

$\max _{\alpha} F_{n}(\alpha, \alpha+\Delta)$ can be obtained when

$$
\begin{gathered}
k \mid \alpha, \quad s k^{2 m-1} \leq \alpha \leq s k^{2 m-1}+k^{m} \quad \text { and } \\
(k-s) k^{2 m-1}-k^{m} \leq \alpha+\Delta \leq(k-s) k^{2 m-1} .
\end{gathered}
$$


Proof. (i) $k \mid \alpha$ follows from Lemma 14 . As $s k^{m-1}<t<(s+1) k^{m-1}$, then

$$
s k^{2 m-1}+k^{m} \leq t k^{m} \leq(s+1) k^{2 m-1}-k^{m}
$$

and using (13.4) one has

$$
k^{2 m}-2(s+1) k^{2 m-1}+k^{m}<\Delta \leq k^{2 m}-2 s k^{2 m-1}-k^{m} .
$$

Now, if $\alpha<s k^{2 m-1}$, then

$$
\alpha+\Delta<k^{2 m}-s k^{2 m-1}-k^{m}=k^{2 m-1}(k-s)-k^{m}
$$

and we apply Lemma 13 . Thus

$$
F_{2 m}\left(s k^{2 m-1}, s k^{2 m-1}+\Delta\right) \geq F_{2 m}(\alpha, \alpha+\Delta) .
$$

If $\alpha>(s+1) k^{2 m-1}$, then $\alpha+\Delta>(k-s-1) k^{2 m-1}+k^{m}$ and we apply the Corollary above. Thus

$$
F_{2 m}\left((s+1) k^{2 m-1},(s+1) k^{2 m-1}+\Delta\right) \geq F_{2 m}(\alpha, \alpha+\Delta) .
$$

If $s k^{2 m-1}<\alpha<(s+1) k^{2 m-1}$, but $(k-s-2) k^{2 m-1}<\alpha+\Delta<(k-s-1) k^{2 m-1}$ or $(k-s) k^{2 m-1}<\alpha+\Delta<(k-s+1) k^{2 m-1}$, then we apply again Lemma 13 or the Corollary, respectively.

The proof of (ii) is analogous.

Proof of Theorem 8. Suppose that $s k^{2 m-1} \leq t k^{m}<(s+1) k^{2 m-1}$. We proceed by induction on $m$ :

$\underline{m=1}:$

$$
k^{2}-2 k t-k \leq \Delta \leq k^{2}-2 k t+k \text {. }
$$

From Lemma 15 (ii) it follows that $\max _{\alpha} F_{2}(\alpha, \alpha+\Delta)$ can be attained, when $\alpha \in\{(t-1) k, t k,(t+1) k\}$. Using (ii) and (vi) of Theorem DKW we verify

$$
\begin{gathered}
F_{2}((t-1) k,(t-1) k+\Delta)=(t-1) k-(t-1)^{2}-\sum_{j=0}^{t-2}\left\lceil\frac{\Delta-j}{k}\right], \\
F_{2}(t k, t k+\Delta)=t k-t^{2}-\sum_{j=0}^{t-1}\left[\frac{\Delta-j}{k}\right], \quad \text { and } \\
F_{2}((t+1) k,(t+1) k+\Delta)=(t+1) k-(t+1)^{2}-\sum_{j=0}^{t}\left\lceil\frac{\Delta-j}{k}\right] .
\end{gathered}
$$

From here, using (13.5), one has

$$
F_{2}(t k, t k+\Delta)-F_{2}((t-1) k,(t-1) k+\Delta)=k-2 t+1-\left\lceil\frac{\Delta-(t-1)}{k}\right\rceil \geq 0
$$

and

$$
F_{2}(t k, t k+\Delta)-F_{2}((t+1) k,(t+1) k+\Delta)=2 t+1-k+\left\lceil\frac{\Delta-t}{k}\right\rceil \geq 0 .
$$

So, $\max _{\alpha} F_{2}(\alpha, \alpha+\Delta)=F_{2}(k t, k t+\Delta)$ for all $\Delta$ satisfying (13.5). 
$\underline{m-1 \rightarrow m}$ : Suppose (i) is true for $m-1$, with respect to any $\Delta_{1}, t_{1}$ satisfying

$$
k^{2 m-2}-2 t_{1} k^{m-1}-k^{m-1} \leq \Delta_{1} \leq k^{2 m-2}-2 t_{1} k^{m-1}+k^{m-1} .
$$

That is

$$
\max _{\alpha} F_{2 m-2}\left(\alpha, \alpha+\Delta_{1}\right)=F_{2 m-2}\left(t_{1} k^{m-1}, t_{1} k^{m-1}+\Delta_{1}\right) .
$$

1) Case $t k^{m}=s k^{2 m-1}, m \geq 2$ :

(To prove this case we do not need to use induction hypothesis.)

a) $k^{2 m}-2 s k^{2 m-1} \leq \Delta \leq k^{2 m}-s k^{2 m-1}+k^{n}$ :

According to Lemma 15 (case (a) in (ii)) it follows that $\max _{\alpha} F_{2 m}(\alpha, \alpha+\Delta)$ can be attained when

$$
k \mid \alpha, \quad s k^{2 m-1}-k^{m} \leq \alpha \leq s k^{2 m-1}, \quad(k-s) k^{2 m-1} \leq \alpha+\Delta \leq(k-s) k^{2 m-1}+k^{m} .
$$

If $\alpha+\Delta=(k-s) k^{2 m-1}$, then we verify (using formula (iv) of Theorem DKW) that

$$
F_{2 m}\left(s k^{2 m-1}, s k^{2 m-1}+\Delta\right)=F_{2 m}\left((k-s) k^{2 m-1}-\Delta,(k-s) k^{2 m-1}\right) .
$$

Let $\alpha+\Delta>(k-s) k^{2 m-1}$ and hence $s k^{2 m-1}-k^{m}<\alpha$. Then

$$
k \mid \alpha ; \quad s k^{2 m-1}-k^{m}<\alpha \leq s k^{2 m-1} ; \quad(k-s) k^{2 m-1}<\alpha+\Delta \leq(k-s) k^{2 m-1}+k^{m}
$$

We compare values $F_{2 m}\left(s k^{2 m-1}, s k^{2 m-1}+\Delta\right)$ and $F_{2 m}(\alpha, \alpha+\Delta)$, where $\alpha$ satisfies (13.6). Using formulas (ii) and (vi) of Theorem DKW one has

$$
\begin{aligned}
F_{2 m}\left(s k^{2 m-1}, s k^{2 m-1}+\Delta\right)= & s k^{2 m-1}-\mu_{k}\left(2 m, s k^{2 m-1}, s k^{2 m-1}+\Delta\right) \\
= & s k^{2 m-1}-\mu_{k}\left(2 m-1, k^{2 m-1}, s k^{2 m-2}+\left\lceil\frac{\Delta-s+1}{k}\right\rceil\right) \\
& -(s-1) s k^{2 m-2}-\sum_{j=0}^{s-2}\left[\frac{\Delta-j}{k}\right\rceil \\
= & s(k-s) k^{2 m-2}-\sum_{j=0}^{s-1}\left[\frac{\Delta-j}{k}\right\rceil
\end{aligned}
$$

and

$$
\begin{aligned}
F_{2 m}(\alpha, \alpha+\Delta)= & \alpha-\mu_{k}(2 m, \alpha, \alpha+\Delta) \\
= & \alpha-\mu_{k}\left(2 m-1, \frac{\alpha}{k}, \alpha+\Delta-(k-s) k^{2 m-1}\right)-(k-s) \frac{\alpha}{k} \\
= & \alpha-\mu_{k}\left(2 m-2, \frac{\alpha}{k}-(s-1) k^{2 m-1}, \frac{\alpha}{k}-(k-s) k^{2 m-2}+\left\lceil\frac{\Delta-s-1}{k}\right\rceil\right) \\
& -(k-s) \frac{\alpha}{k}-(s-1) \frac{\alpha}{k}+(s-1)(k-s) k^{2 m-2}-\sum_{j=0}^{s-2}\left\lceil\frac{\Delta-j}{k}\right\rceil
\end{aligned}
$$




$$
\begin{aligned}
= & \frac{\alpha}{k}+(s-1)(k-s) k^{2 m-2}-\sum_{j=0}^{s-2}\left\lceil\frac{\Delta-j}{k}\right\rceil \\
& -\mu_{k}\left(2 m-2, \frac{\alpha}{k}-(s-1) k^{2 m-1}, \frac{\alpha}{k}-(k-s) k^{2 m-2}+\left\lceil\frac{\Delta-s+1}{k}\right\rceil\right) .
\end{aligned}
$$

From (13.6) if follows that $k^{2 m-2}-k^{m-1}<\frac{\alpha}{k}-(s-1) k^{2 m-1} \leq k^{2 m-2}$ and $0<\frac{\alpha}{k}-(k-s) k^{2 m-2}+\left\lceil\frac{\Delta-s+1}{k}\right\rceil \leq k^{m-1}$. Let us show that for all $\gamma, \delta$ such that $k^{2 m-2}-k^{m-1}<\gamma \leq k^{2 m-2}, 0<\delta \leq k^{m-1}$ it is true that $\mu_{k}(2 m-2, \gamma, \delta)=\delta$. It is known that $\mu_{k}(2 m-2, \gamma, \delta) \geq \frac{\gamma \cdot \delta}{k^{2 m-2}}$ implies $\mu_{k}(2 m-2, \gamma, \delta) \geq \frac{\gamma \cdot \delta}{k^{2 m-2}}>$ $\frac{\left(k^{2 m-2}-k^{m-1}\right) \delta}{k^{2 m-2}}=\delta-\frac{\delta}{k^{m-1}} \geq \delta-1$. So, $\delta \geq \mu_{k}(2 m-2, \gamma, \delta)>\delta-1$ implies $\mu_{k}(2 m-2, \gamma, \delta)=\delta$. Therefore, $\mu\left(2 m-2, \frac{\alpha}{k}-(s-1) k^{2 m-1}, \frac{\alpha}{k}-(k-s) k^{2 m-2}+\right.$ $\left.\left\lceil\frac{\Delta-s+1}{k}\right\rceil\right)=\frac{\alpha}{k}-(k-s) k^{2 m-2}+\left\lceil\frac{\Delta-s+1}{k}\right\rceil$ and hence for all $\alpha$ satisfying (13.6) one has

$$
F_{2 m}(\alpha, \alpha+\Delta)=s(k-s) k^{2 m-2}-\sum_{j=0}^{s-1}\left\lceil\frac{\Delta-j}{k}\right\rceil=F_{2 m}\left(s k^{2 m-1}, s k^{2 m-1}+\Delta\right) .
$$

b) $k^{2 m}-2 s k^{2 m-1}-k^{m} \leq \Delta \leq k^{2 m}-2 s k^{2 m-1}$ :

This case can be treated analogously. So, if $t k^{m}=s k^{2 m-1}$, then

$$
\max _{\alpha} F_{2 m}(\alpha, \alpha+\Delta)=F_{2 m}\left(s k^{2 m-1}, s k^{2 m-1}+\Delta\right) \text {. }
$$

2) Case $s k^{2 m-1}<t k^{m}<(s+1) k^{2 m-1}, m \geq 2$ :

Using again formula (ii) of Theorem DKW one has

$$
\begin{aligned}
F_{2 m} & \left(t k^{m}, t k^{m}+\Delta\right) \\
= & t k^{m}-\mu_{k}\left(2 m, t k^{m}, t k^{m}+\Delta\right) \\
= & t k^{m}-(k-s-1) t k^{m-1}-\mu_{k}\left(2 m-1, t k^{m-1}, t k^{m}+\Delta-(k-s-1) k^{2 m-1}\right) \\
= & t k^{m}-(k-s-1) t k^{m-1}-s t k^{m-1}+s(k-s-1) k^{2 m-2}-\sum_{j=0}^{s-1}\left[\frac{\Delta-j}{k}\right\rceil \\
& \quad-\mu_{k}\left(2 m-2, t k^{m-1}-s k^{2 m-2}, t k^{m-1}-(k-s-1) k^{2 m-2}+\left\lceil\frac{\Delta-s}{k}\right\rceil\right) \\
= & k^{m-1} t+s k^{2 m-2}(k-s-1)-\sum_{j=0}^{s-1}\left\lceil\frac{\Delta-j}{k}\right\rceil \\
& \quad-\mu_{k}\left(2 m-2, t k^{m-1}-s k^{2 m-2}, t k^{m-1}-(k-s-1) k^{2 m-2}+\left\lceil\frac{\Delta-s}{k}\right\rceil\right) .
\end{aligned}
$$


Lemma 15 says that in this case $\max _{\alpha} F_{2 m}(\alpha, \alpha+\Delta)$ can be attained, when $k \mid \alpha, \quad s k^{2 m-1} \leq \alpha \leq(s+1) k^{2 m-1}$ and $(k-s-1) k^{2 m-1} \leq \alpha+\Delta \leq(k-s) k^{2 m-1}$. At first let us consider the extremal cases, that is $\left(^{*}\right) \alpha=s k^{2 m-1}$ and $\left({ }^{* *}\right) \alpha+\Delta=$ $(k-s-1) k^{2 m-1}$.

$\left(^{*}\right) \alpha=s k^{2 m-1}$ : Using (13.7) and (13.8) one has $F_{2 m}\left(t k^{m}, t k^{m}+\Delta\right)-F_{2 m}\left(s k^{2 m-1}\right.$, $\left.s k^{2 m-1}+\Delta\right)=k^{m-1} t-s k^{2 m-2}-\mu_{k}\left(2 m-2, k^{m-1} t-s k^{2 m-2}, k^{m-1} t \quad-\right.$ $\left.(k-s-1) k^{2 m-2}+\left\lceil\frac{\Delta-s}{k}\right\rceil\right) \geq 0$, since $\mu_{k}(m, \gamma, \delta) \leq \min (\gamma, \delta)$ for all $\gamma, \delta$.

${ }^{(* *)} \alpha+\Delta=(k-s-1) k^{2 m-1}$ : As $k \mid \alpha$, we have $k \mid \Delta$ and hence

$$
\begin{aligned}
F_{2 m}\left(t k^{m}, t k^{m}+\Delta\right)= & k^{m-1}+s k^{2 m-2}(k-s-1)-\frac{s \Delta}{k} \\
& -\mu_{k}\left(2 m-2, k^{m-1} t-s k^{2 m-2}, k^{m-1} t-(k-s-1) k^{2 m-2}+\frac{\Delta}{k}\right)
\end{aligned}
$$

and we verify that

$$
\begin{aligned}
& F_{2 m}\left((k-s-1) k^{2 m-1}-\Delta,(k-s-1) k^{2 m-1}\right) \\
& =(k-s-1) k^{2 m-2} \cdot(s+1)-\frac{\Delta(s+1)}{k} .
\end{aligned}
$$

\section{Therefore}

$$
\begin{aligned}
& F_{2 m}\left(t k^{m}, t k^{m}+\Delta\right)-F_{2 m}\left((k-s-1) k^{2 m-1}-\Delta,(k-s-1) k^{2 m-1}\right) \\
& =k^{m-1} t-k^{2 m-2}(k-s-1)+\frac{\Delta}{k} \\
& \quad-\mu_{k}\left(2 m-2, k^{m-1} t-s k^{2 m-2}, k^{m-1} t-k^{2 m-2}(k-s-1)+\frac{\Delta}{k}\right) \geq 0 .
\end{aligned}
$$

Now let

$k \mid \alpha, \quad s k^{2 m-1}<\alpha \leq(s+1) k^{2 m-1}$ and $(k-s-1) k^{2 m-1}<\alpha+\Delta \leq(k-s) k^{2 m-1}$.

Then (we use (ii) of Theorem DKW)

$$
\begin{aligned}
F_{2 m}(\alpha, \alpha+\Delta)= & \frac{\alpha}{k}+s k^{2 m-2}(k-s-1) \\
& -\mu_{k}\left(2 m-2, \frac{\alpha}{k}-s k^{2 m-2}, \frac{\alpha}{k}-(k-s-1) k^{2 m-2}+\left\lceil\frac{\Delta-s}{k}\right\rceil\right) \\
& -\sum_{j=0}^{s-1}\left\lceil\frac{\Delta-j}{k}\right\rceil .
\end{aligned}
$$

Now we compare the values $F_{2 m}\left(t k^{m}, t k^{m}+\Delta\right)$ and $F_{2 m}(\alpha, \alpha+\Delta)$. One has

$$
F_{2 m}\left(t k^{m}, t k^{m}+\Delta\right)-F_{2 m}(\alpha, \alpha+\Delta)=t k^{m-1}-\frac{\alpha}{k}-\mu_{(1)}+\mu_{(2)},
$$


where

$$
\begin{aligned}
& \mu_{(1)}=\mu_{k}\left(2 m-2, k^{m-1} t-s k^{2 m-2}, k^{m-1} t-(k-s-1) k^{2 m-2}+\left\lceil\frac{\Delta-s}{k}\right\rceil\right) \\
& \mu_{(2)}=\mu_{k}\left(2 m-2, \frac{\alpha}{k}-s k^{2 m-2}, \frac{\alpha}{k}-(\dot{k}-s-1) k^{2 m-2}+\left\lceil\frac{\Delta-s\rceil}{k}\right\rceil\right) .
\end{aligned}
$$

We use abbreviations $t_{1}=t-s k^{m-1}$ and $\alpha_{1}=\frac{\alpha}{k}-s k^{2 m-2}$. Then $\mu_{(1)}=$ $\mu_{k}\left(2 m-2, k^{m-1} t_{1}, k^{m-1} t_{1}+\Delta_{1}\right)$ and $\mu_{(2)}=\mu_{k}\left(2 m-2, \alpha_{1}, \alpha_{1}+\Delta_{1}\right)$, where $\Delta_{1}=$ $(2 s+1-k) k^{2 m-2}+\left\lceil\frac{\Delta-s}{k}\right\rceil$. We verify that

$$
k^{2 m-2}-2 t_{1} k^{m-1}-k^{m-1} \leq \Delta_{1} \leq k^{2 m-2}-2 t_{1} k^{m-1}+k^{m-1} .
$$

(13.10) can be written in the form:

$$
\begin{aligned}
F_{2 m}\left(t k^{m}, t k^{m}+\Delta\right)-F_{2 m}(\alpha, \alpha+\Delta)= & F_{2 m-2}\left(t_{1} k^{m-1}, t_{1} k^{m-1}+\Delta_{1}\right) \\
& -F_{2 m-2}\left(\alpha_{1}, \alpha_{1}+\Delta_{1}\right) \geq 0,
\end{aligned}
$$

according to the induction hypothesis. We remark that (ii) can be proved in the same way.

Finally we introduce now some simplification for the determination of the value $F_{2 m}\left(t k^{m}, t k^{m}+\Delta\right)$. If $\Delta$ satisfies (i), then

$$
t k^{m}+\Delta=k^{2 m}-k^{m} t+\delta \quad \text { for some } \quad-k^{m} \leq \delta \leq k^{m} .
$$

\section{Lemma 16.}

a) If $-k^{m} \leq \delta \leq 0$, then

$$
F_{2 m}\left(t k^{m}, t k^{m}+\Delta\right)=t^{2}+t-\mu_{k}\left(m, t, k^{m}+\delta\right)
$$

(b) If $0 \leq \delta \leq k^{m}$, then

$$
F_{2 m}\left(t k^{m}, t k^{m}+\Delta\right)=t^{2}-\mu_{k}(m, t, \delta) .
$$

Proof. a) $-k^{m} \leq \delta \leq 0$ :

Let $t k^{m}=s_{1} k^{2 m-1}+s_{2} k^{2 m-2}+\cdots+s_{m} k^{m}$. Then

$$
t k^{m}+\Delta=k^{2 m}-k^{m} t+\delta=\left(k-s_{1}-1\right) k^{2 m-1}+\cdots+\left(k-s_{m}-1\right) k^{m}+k^{m}+\delta \text {. }
$$

We use again formula (ii) of Theorem DKW. One has

$$
\begin{aligned}
& \mu_{k}\left(2 m, t k^{m},(k-s-1) k^{2 m-1}+\cdots+\left(k-s_{m}-1\right) k^{m}+k^{m}+\delta\right) \\
& =\mu_{k}\left(2 m-1, k^{m-1} t,\left(k-s_{2}-1\right) k^{2 m-2}+\cdots+k^{m}+\delta\right)+\cdots \\
& \quad+\left(k-s_{1}-1\right) k^{m-1} t=\cdots=\mu_{k}\left(m, t, k^{m}+\delta\right)+\sum_{i=1}^{m}\left(k-s_{i}-1\right) k^{m-i} t \\
& =\mu_{k}\left(m, t, k^{m}+\delta\right)+k^{m} t-t-t \sum_{i=1}^{m} s_{i} k^{m-i}=\mu_{k}\left(m, t, k^{m}+\delta\right)+k^{m} t-t-t^{2},
\end{aligned}
$$


because $\sum_{i=1}^{m} s_{i} k^{m-i}=t$. Therefore we have

$$
F_{2 m}\left(t k^{m}, t k^{m}+\Delta\right)=t k^{m}-\mu_{k}\left(t k^{m}, t k^{m}+\Delta\right)=t^{2}+t-\mu_{k}\left(m, t, k^{m}+\delta\right) .
$$

(b) is proved analogously.

\section{On Optimal and Bisaturated Pairs}

Recall that $(A, B) \in \mathscr{C} \mathscr{A}_{\mathscr{C}} \mathscr{C}_{k}(n)$ is called optimal exactly if $|B|=f_{n}(|A|)$. An optimal pair $(A, B)$ is always $A$-saturated, but not necessarily $B$-saturated. We try to describe pairs of cardinalities $(|A|,|B|)$ for all pairs $(A, B)$, which are optimal and bisaturated. Earlier we denoted this set by $\mathscr{M}(n)=\mathscr{O}(n) \cap \mathscr{S}(n)$. Clearly for $(A, B) \in$ $\mathscr{C} \mathscr{A} \mathscr{C}_{k}(n)$

$$
(A, B) \in \mathscr{M}(n) \quad \text { iff } \quad f_{n}\left(f_{n}(|A|)\right)=|A| .
$$

We also know (see Corollary (iii) in Section 7), that for all $\alpha$

$$
f_{n}\left(f_{n}\left(f_{n}(a)\right)\right)=f_{n}(a)
$$

and hence for all $a$

$$
(C, D) \in \mathcal{O}(n), \quad|C|=f_{n}(a), \quad|D|=f_{n}\left(f_{n}(a)\right) \quad \text { implies } \quad(C, D) \in \mathscr{M}(n) .
$$

Theorem 9. Let $(A, B) \in \mathcal{O}(n),|A|=a,|B|=f_{n}(a)$. Then

(i) $(A, B) \in \mathscr{M}(n)$ implies $k !(|A|-|B|)$.

(ii) For all $a \leq k^{n-2}(A, B) \in \mathscr{M}(n)$.

(iii) For all $a, k^{n-2} \leq a \leq k^{n-2}(k-1)^{2},(A, B) \in \mathscr{M}(n)$ iff $k \mid(|A|-|B|)$.

(iv) For any integer $\Delta ;-\left(k^{n}-s k^{n-1}\right) \leq \Delta \leq k^{n}-2 k^{n-1}$; with $k \mid \Delta$ there is a unique $a ; k^{n-2} \leq a \leq(k-1)^{2} k^{n-2}$ for which $(A, B) \in \mathscr{M}(n)$,

$$
|A|=a, \quad|B|=f_{n}(a) \quad \text { and } \quad|B|-|A|=\Delta .
$$

(v) Let $M(n)$ equal the number of different pairs of integers $(|A|,|B|)$, where $|A| \leq|B|$ and $(A, B) \in \mathscr{M}(n)$. Then

$$
M(n)=k^{n-1}-k^{n-2}+1 .
$$

At first let us introduce an auxiliary result.

Lemma 17. Let $k^{n-2} \leq a \leq(k-1)^{2} k^{n-1}$ and let

$$
\alpha-\mu_{k}(n, \alpha, \beta)=a, \quad \beta-\mu_{k}(n, \alpha, \beta)=f_{n}(a) .
$$

Then $k^{n-1} \leq \alpha, \beta \leq(k-1) k^{n-1}$.

Proof. We know that

$$
f_{n}\left(k^{n-2}\right)=(k-1)^{2} k^{n-2} \quad \text { (follows from Theorem 3) }
$$

and that $(A, B) \in \mathscr{M}(n)$, where $|A|=k^{n-2},|B|=(k-1)^{2} k^{n-2}$. Therefore, for any $a$, 
$k^{n-2} \leq a \leq(k-1)^{2} k^{n-2}$,

$$
\left|f_{n}(a)-a\right| \leq k^{n}-2 k^{n-1}=f_{n}\left(k^{n-2}\right)-k^{n-2} .
$$

Suppose, for some $a, k^{n-2} \leq a \leq f_{n}(a) \leq(k-1)^{2} k^{n-2}, \alpha<k^{n-1}$. We denote $\Delta_{a}=\beta-\alpha$ and together with (14.4) let us consider

$$
k^{n-1}-\mu_{k}\left(n, k^{n-1}, k^{n-1}+\Delta_{a}\right), \quad k^{n-1}+\Delta_{a}-\mu_{k}\left(n, k^{n-1}, k^{n-1}+\Delta_{a}\right) .
$$

As $\alpha<k^{n-1}, \Delta_{a}=f_{n}(a)-a \leq k^{n}-2 k^{n-1}$, then $k^{n-1}+\Delta_{a} \leq(k-1) k^{n-1}$. Therefore, from Lemma 13 it follows that

$$
\begin{gathered}
\quad k^{n-1}-\mu_{k}\left(n, k^{n-1}, k^{n-1}+\Delta_{a}\right) \geq \alpha-\mu_{k}\left(n, \alpha, \alpha+\Delta_{a}\right)=\alpha-\mu_{k}(n, \alpha, \beta)=a \\
\Delta_{a}+k^{n-1}-\mu_{k}\left(n, k^{n-1}, k^{n-1}+\Delta_{a}\right) \geq \beta-\mu_{k}\left(n, \alpha, \alpha+\Delta_{a}\right)=f_{n}(a) \\
\text { Now, if } k^{n-1}-\mu_{k}\left(n, k^{n-1}, k^{n-1}+\Delta_{a}\right)=a_{1}>a \text {, then } \\
f_{n}\left(a_{1}\right) \geq k^{n-1}+\Delta_{a}-\mu_{k}\left(n, k^{n-1}, k^{n-1}+\Delta_{a}\right)>f_{n}(a) .
\end{gathered}
$$

This is a contradiction, since $f_{n}(\cdot)$ is a monotonically decreasing function.

Hence

$$
\begin{aligned}
k^{n-1}-\mu_{k}\left(n, k^{n-1}, k^{n-1}+\Delta_{a}\right) & =a, \\
k^{n-1}+\Delta_{a}-\mu_{k}\left(n, k^{n-1}, k^{n-1}+\Delta_{a}\right) & =f_{n}(a) .
\end{aligned}
$$

Suppose that $\Delta_{a}=k \cdot r+\Delta_{1}, 0 \leq \Delta_{1}<k$.

If 1$) 0<\Delta_{1}<k$, we verify (using (ii) of Theorem $\mathrm{DKW}$ ) that

$$
\mu_{k}\left(n, k^{n-1}, k^{n-1}+\Delta_{a}\right)=\left\lceil\frac{k^{n-1}+\Delta_{a}}{k}\right\rceil=\mu_{k}\left(n, k^{n-1}, k^{n-1}+\Delta_{a}+k-\Delta_{1}\right)
$$

and hence

$$
\begin{gathered}
k^{n-1}-\mu_{k}\left(n, k^{n-1}, k^{n-1}+\Delta_{a}+k-\Delta_{1}\right)=a, \\
k^{n-1}+\Delta_{a}+k-\Delta_{1}-\mu_{k}\left(n, k^{n-1}, k^{n-1}+\Delta_{a}+k-\Delta_{1}\right)=f_{n}(a)+k-\Delta_{1}>f_{n}(a),
\end{gathered}
$$

a contradiction.

If 2) $\Delta_{a}=0$, then, as $\mu_{k}(n, \alpha, \beta) \geq \frac{\alpha \beta}{k^{n}}$, one has $\alpha-\mu_{k}\left(n, \alpha, \alpha+\Delta_{a}\right) \leq \alpha-$ $\frac{\alpha\left(\alpha+\Delta_{a}\right)}{k^{n}}=T\left(\alpha, \Delta_{a}\right)$, say, and we verify that $T\left(\alpha^{\prime}, \Delta_{a}\right)<T\left(\alpha^{\prime \prime}, \Delta_{a}\right)$, if $\alpha^{\prime}<\alpha^{\prime \prime} \leq$ $k^{n-1} \leq \frac{k^{n}-\Delta_{a}}{2}$. Therefore $\alpha-\mu_{k}\left(n, \alpha, \alpha+\Delta_{a}\right) \leq T\left(\alpha, \Delta_{a}\right)<T\left(k^{n-1}, \Delta_{a}\right)=k^{n-1}-$ $\mu_{k}\left(n, k^{n-1}, k^{n-1}+\Delta_{a}\right)$ and since $k \mid \Delta_{a}$ we have $\mu_{k}\left(n, k^{n-1}, k^{n-1}+\Delta\right)=\frac{k^{n-1}\left(k^{n-1}+\Delta\right)}{k^{n}}$. Hence $a=\alpha-\mu_{k}\left(n, \alpha, \alpha+\Delta_{a}\right)<k^{n-1}-\mu_{k}\left(n, k^{n-1}, k^{n-1}+\Delta_{a}\right)$ and this is also a contradiction, because we assumed (14.6). $\alpha, \beta \leq(k-1) k^{n-1}$ is proved analogously. 


\section{Proof of Theorem 9.}

(i) This restates Theorem 5 .

(ii) Let us show that for all $a<k^{n-2} f_{n}(a)>f_{n}(a+1)$, from where the claim follows. We use our main recursive formula: for $a \leq k^{n-2}$

$$
f_{n}(a)=(k-1) k^{n-1}+k f_{n-2}(a)-(k-1) a .
$$

Hence, as $f_{n-2}(a) \geq f_{n-2}(a+1)$

$$
\begin{aligned}
f_{n}(a) \geq & (k-1) k^{n-1}+k f_{n-2}(a+1)-(k-1) a>(k-1) k^{n-1} \\
& +k f_{n-2}(a+1)-(k-1)(a+1)=f_{n}(a+1) .
\end{aligned}
$$

(iii) Follows from (i) and (iv).

(iv) Let $\mathscr{M}_{1}(n) \subset \mathscr{M}(n)$ be defined by

$$
\begin{aligned}
\mathscr{M}_{1}(n) & =\left\{\left(A_{i}, B_{i}\right)_{i=1}^{N}:\left(A_{i}, B_{i}\right) \in \mathscr{M}(n), k^{n-2}=\left|A_{1}\right|<\left|A_{2}\right|<\cdots<\left|A_{N}\right|\right. \\
& \left.=(k-1)^{2} k^{n-2} ;(k-1) k^{n-2}=\left|B_{1}\right|>\left|B_{2}\right|>\cdots>\left|B_{N}\right|=k^{n-2}\right\} .
\end{aligned}
$$

Obviously, $\left|A_{i}\right|=\left|B_{N-i}\right|$.

We show that for all $1 \leq i<N\left|A_{i+1}\right|-\left|A_{i}\right|<k$ (or equivalently for all $1 \leq j<N\left|B_{j}\right|-\left|B_{j+1}\right|<k$ ), from where, using (i), (iv) follows. Assume it does not and there exists $i, 1 \leq i<N$ for which $\left|A_{i+1}\right|-\left|A_{i}\right| \geq k$. Suppose that for some $\alpha, \beta$

$$
\alpha-\mu_{k}(n, \alpha, \beta)=\left|A_{i+1}\right| \quad \text { and } \quad \beta-\mu_{k}(n, \alpha, \beta)=\left|B_{i+1}\right|=f_{n}\left(\left|A_{i+1}\right|\right) \text {. }
$$

According to Lemma $17 k^{n-1} \leq \alpha, \beta \leq(k-1) k^{n-1}$. Also, as $\left(A_{i+1}, B_{i+1}\right) \in \mathscr{M}(n)$, then $k \mid \alpha$ and $k \mid \beta$.

Now, we consider

$$
\alpha-k-\mu_{k}(n, \alpha-k, \beta)=\alpha^{*} \quad \text { and } \quad \beta-\mu_{k}(n, \alpha-k, \beta)=b .
$$

Let us show that $\mu_{k}(n, \alpha-k, \beta)<\mu_{k}(n, \alpha, \beta)$ for $\beta \geq k^{n-1}, k|\alpha, k| \beta$.

If $\beta=k^{n-1}$, then using (ii) of Theorem DKW one has $\mu_{k}\left(n, \alpha-k, k^{n-1}\right)=$ $\mu_{k}\left(n-1, \frac{\alpha}{k}-1, k^{n-1}\right)=\frac{\alpha}{k}-1=\mu_{k}\left(n, \alpha, k^{n-1}\right)-1<\mu_{k}\left(n, \alpha, k^{n-1}\right)$. Let $s k^{n-1}<$ $\beta \leq(s+1) k^{n-1}, s>0$. Then $\mu_{k}(n, \alpha-k, \beta)=\mu_{k}\left(n-1, \frac{\alpha}{k}-1, \beta-s k^{n-1}\right)+$ $s\left(\frac{\alpha}{k}-1\right) \leq \mu_{k}\left(n-1, \frac{\alpha}{k}, \beta-s k^{n-1}\right)+s \frac{\alpha}{k}-s=\mu_{k}(n, \alpha, \beta)-s<\mu_{k}(n, \alpha, \beta)$.

Therefore,

$$
a^{*}=\alpha-k-\mu_{k}(n, \alpha-k, \beta)>\alpha-k-\mu_{k}(n, \alpha, \beta)=\left|A_{i+1}\right|-k \geq\left|A_{i}\right|
$$

and

$$
f\left(a^{*}\right) \geq b=\beta-\mu_{k}(n, \alpha-k, \beta)>\beta-\mu_{k}(n, \alpha, \beta)=f\left(A_{i+1}\right) .
$$

We consider $\left(A^{*}, B^{*}\right) \in \mathscr{M}(n)$, where $\left|A^{*}\right|=f_{n}\left(f_{n}\left(a^{*}\right)\right), \quad\left|B^{*}\right|=f_{n}\left(a^{*}\right)$. Now $\left|A^{*}\right|<\left|A_{i+1}\right|$ is impossible, since $\left|A^{*}\right|=f_{n}\left(f_{n}\left(a^{*}\right)\right) \geq a^{*}>\left|A_{i}\right|$ and hence the next 
pair of $\left(A_{i}, B_{i}\right)$ is not $\left(A_{i+1}, B_{i+1}\right)$. Also $\left|A^{*}\right| \geq\left|A_{i+1}\right|$ is impossible, since $\left|B^{*}\right|=$ $f_{n}\left(a^{*}\right)>f\left(A_{i+1}\right)$ and hence $\left(A_{i+1}, B_{i+1}\right) \notin \mathscr{M}(n)$. Finally, (v) follows from (ii) and (iv),

Now for all $\Delta ;-\left(k^{n}-2 k^{n-1}\right) \leq \Delta \leq k^{n}-2 k^{n-1}$; and $\Delta \mid k$ let us find $(A, B) \epsilon$ $\mathscr{M}_{1}(n)$ for which $|B|=|A|+\Delta$. From the definition of $F_{n}(\alpha, \alpha+\Delta)$ it follows that for all $0 \leq a \leq k^{n}$

$$
\max _{\alpha} F_{n}\left(\alpha, \alpha+f_{n}(a)-a\right)=a .
$$

We formulate a consequence of Theorems 8,9 and Lemma 17.

Theorem 10. For $n=2 m$ (the case $n=2 m+1$ is similar $)$

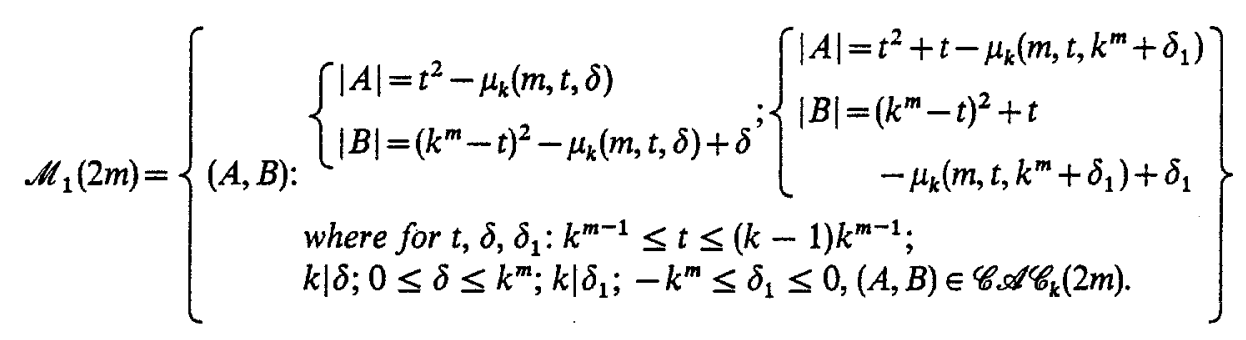

\section{Explicit Values of $f_{a}(a)$}

We return to our original question: what is $f_{n}(a)$ ?

\section{Theorem 11.}

(i) $f_{2 m}\left(t^{2}\right)=\left(k^{m}-t\right)^{2}$, for all $0 \leq t \leq k^{m}$.

(ii) $f_{2 m}\left(t^{2}-t\right)=\left(k^{m}-t\right)\left(k^{m}-t+1\right)$, for all $1<t$.

(iii) $f_{2 m}\left(\left(s k^{m-1}\right)^{2}-\frac{s \delta}{k}\right)=\left(k^{m}-s k^{m-1}\right)^{2}+\frac{\delta(k-s)}{k}$ for all $0<s<k ; k \mid \delta ;-k^{m} \leq$ $\delta \leq k^{m}$.

Moreover, all pairs $(A, B)$ with above mentioned parameters are optimal and bisaturated.

Proof. (i) equals (i) of Theorem 3. It can also be proved inductively on $m$ as the case (ii) to follow.

(ii) If a) $k^{m-1} \leq t \leq(k-1) k^{m-1}$, then we apply Theorem 10 . We put $\delta=k^{m}$ and get

$$
\begin{gathered}
|A|=t^{2}-\mu_{k}\left(m, t, k^{m}\right)=t^{2}-t, \\
|B|=\left(k^{m}-t\right)^{2}-\mu_{k}\left(m, t, k^{m}\right)+k^{m}=\left(k^{m}-t\right)\left(k^{m}-t+1\right)
\end{gathered}
$$

and as $(A, B) \in \mathscr{M}(2 m)$, then $f_{2 m}\left(t^{2}-t\right)=\left(k^{m}-t\right)\left(k^{m}-t+1\right)$.

If b) $(k-1) k^{m-1}<t<k^{m}$, then we abbreviate $t_{1}=k^{m}-t+1 ; 1<t_{1} \leq k^{m-1}$, and consider $f_{2 m}\left(t_{1}^{2}-t_{1}\right)$. Now, if $f_{2 m}\left(t_{1}^{2}-t_{1}\right)=\left(k^{m}-t_{1}\right)\left(k^{m}-t_{1}+1\right)=t^{2}-t$, 
then $f_{2 m}\left(t^{2}-t\right)=t_{1}^{2}-t_{1}=\left(k^{m}-t\right)\left(k^{m}-t+1\right)$ according to (ii) of Theorem 9. Therefore it is sufficient to consider

c) $1<t<k^{m-1}$ :

We proceed by induction on $m$.

$\underline{m=1}$ : As $1<t<k$ (condition of (ii), then $t$ is from the interval a) which is settled.

$\underline{m-1 \rightarrow m:}$

$$
f_{2 m-2}\left(t^{2}-t\right)=\left(k^{m-1}-t\right)\left(k^{m-1}-t+1\right) \quad \text { for all } 1<t<k^{m-1} .
$$

Now we use the induction hypothesis (15.1) and our recursive formula for $1<t<k^{m-1}$ :

$$
\begin{aligned}
f_{2 m}\left(t^{2}-t\right) & =(k-1) k^{2 m-1}+k f_{2 m-2}\left(t^{2}-t\right)-(k-1)\left(t^{2}-t\right) \\
& =(k-1) k^{2 m-1}+k\left(k^{m-1}-t\right)\left(k^{m-1}-t+1\right)-(k-1)\left(t^{2}-t\right) \\
& =\left(k^{2}-t\right)\left(k^{m}-t+1\right) .
\end{aligned}
$$

(iii) follows from Theorem 10. We put $t=s k^{m-1}$ and verify that for $0 \leq \delta \leq k^{m}$, $k \mid \delta$

$$
\begin{aligned}
& |A|=\left(s k^{m-1}\right)^{2}-\mu_{k}\left(m, s k^{m-1}, \delta\right)=\left(s k^{m-1}\right)-\frac{s \delta}{k}, \\
& |B|=\left(k^{m}-s k^{m-1}\right)^{2}+\frac{\delta(k-s)}{k}
\end{aligned}
$$

and for $-k^{m} \leq \delta_{1} \leq 0, m \mid \delta_{1}$

$$
\begin{aligned}
& |A|=\left(s k^{m-1}\right)^{2}+s k^{m-1}-\frac{s\left(k^{m}+\delta_{1}\right)}{k}=\left(s k^{m-1}\right)^{2}-\frac{s \delta_{1}}{k}, \\
& |B|=\left(k^{m}-s k^{m-1}\right)^{2}+\frac{\delta_{1}(k-s)}{k}
\end{aligned}
$$

and so (iii) holds.

Remark. For $n$ odd there are similar results.

\section{An Algorithm for Computing $f_{n}(a)$}

We distinguish three cases

Case. $a \leq k^{n-2}$ :

Here we apply our recursive formula.

Case. $k^{n-2} \leq a \leq(k-1)^{2} k^{n-2}$ :

We do the case $n=2 m$ (the case $n$ odd is similar). We find the unique $t$ for which $t^{2}-t<a \leq t^{2}+t ; k^{m-1} \leq t \leq(k-1) k^{m-1}$. 
Subcase 1) $t^{2}-t<a \leq t^{2}$ :

We find the unique $\delta, 0 \leq \delta<k^{m}, k \mid \delta$ for which $t^{2}-\mu_{k}(m, t, \delta+k)<a \leq t^{2}-$ $\mu_{k}(m, t, \delta)$. Then $f_{2 m}(a)=\left(k^{m}-t\right)^{2}-\mu_{k}(m, t, \delta)+\delta$.

Subcase 2) $t^{2}<a \leq t^{2}+t$ :

We find the unique $\delta_{1}, k \mid \delta_{1} ;-k^{m} \leq \delta_{1}<0$ for which $t^{2}+t-\mu_{k}\left(m, t, k^{m}+\delta_{1}+k\right)$ $<a \leq t^{2}+t-\mu_{k}\left(m, t, k^{m}+\delta_{1}\right)$. Then $f_{2 m}(a)=\left(k^{m}-t\right)^{2}+t-\mu_{k}\left(m, t, k^{m}+\delta_{1}\right)+$ $\delta_{1}$.

Let us note that, as $\mu_{\mathrm{k}}(m, t, \delta)$ is a strictly monotonically increasing function in $\delta$ for any fixed $t ; k^{m-1} \leq t \leq(k-1) k^{m-1}$; and also note that $k \mid \delta$. Therefore one needs to find $\delta$ by at most $\log _{2} k^{m-1}=(m-1) \log _{2} k$ trials.

Case. $a>(k-1)^{2} k^{2 m-2}$ :

We find the unique $t ; t \geq(k-1) k^{m-1}$ for which $t^{2}-t<a \leq t^{2}+t$.

Subcase 1) $t^{2}<a \leq t^{2}+t$ :

According to (ii) of Theorem 11 one has

$$
\left(k^{m}-t\right)\left(k^{m}-t-1\right) \leq f_{2 m}(a)<\left(k^{m}-t\right)^{2} .
$$

We find the unique $b ;\left(k^{m}-t\right)\left(k^{m}-t-1\right) \leq b<\left(k^{m}-t\right)^{2}$, for which

$$
f_{2 m}(b+1)<a \leq f_{2 m}(b) \text {. }
$$

Then $f_{2 m}(a)=b$.

Subcase 2) $t^{2}-t<a \leq t^{2}$ :

Here $\left(k^{m}-t\right)^{2} \leq f_{2 m}(a)<\left(k^{m}-t\right)\left(k^{m}-t+1\right)$ and we find $b_{1},\left(k^{m}-t\right)^{2} \leq b_{1}<$ $\left(k^{m}-t\right)\left(k^{m}-t+1\right)$, for which $f_{2 m}\left(b_{1}+1\right)<a \leq f_{2 m}\left(b_{1}\right)$. Then

$$
f_{2 m}(a)=b_{1} \text {. }
$$

Acknowledgement. The authors thank George F. Clements for his admirable efforts in helping to improve the presentation.

\section{References}

1. Ahlswede, R., Zhang, Z:: On cloud-antichains and related configurations. Discrete Math. 85, 225-245 (1990)

2. Seymour, P.: On incomparable families of sets. Mathematica 20, 208-209 (1973)

3. Kleitman, D.J.: Families on non-disjoint subsets. J. Comb. Theory 1, 153-155 (1966)

4. Hilton, A.J.: A theorem on finite sets. Quart. J. Math. Oxford (2), 27, 33-36 (1976)

5. Daykin, D.E., Kleitman, D.J., West, D.B.: The number of meets between two subsets of a lattice. J. Comb. Theory, Ser. A 26, 135-156 (1979)

6. Ahlswede, R., Daykin, D.E.: An inequality for the weights of two families of sets, their unions and intersections. Z. Wahrscheinlichkeitstheorie u. verw. Geb. 43, 183-185 (1978)

7. Ahlswede, R., Zhang, Z.: A new direction in extremal theory. J. Comb. Inf. \& Syst. Sc. (to be published) 
8. Ahlswede, R., Khachatrian, L.H.: The maximal length of cloud-antichains. SFB 343, Preprint 91-116, Discrete Math. 131, 9-15 (1994)

9. Ahlswede, R., Khachatrian, L.H.: Sharp bounds for cloud-antichains of length two, SFB 343, Preprint 92-012

10. Ahlswede, R., Khachatrian, L.H.: Towards equality characterisation in correlation inequalities, SFB 343 Preprint. the Europ. J. Comb. (to be published)

Received: August 8, 1993

Revised: March 31, 1995 